\title{
OPEN Experimental adaptation of dengue virus 1 to Aedes albopictus mosquitoes by in vivo selection
}

\author{
Rachel Bellone ${ }^{1,2,8}$, Sebastian Lequime ${ }^{2,3,4,8}$, Henri Jupille 1,8, Giel P. Göertz ${ }^{5}$, Fabien Aubry ${ }^{3}$, \\ Laurence Mousson ${ }^{1}$, Géraldine Piorkowski ${ }^{6}$, Pei-Shi Yen ${ }^{1}$, Gaelle Gabiane ${ }^{1}$, Marie Vazeille ${ }^{1}$, \\ Anavaj Sakuntabhai ${ }^{7}$, Gorben P. Pijlman ${ }^{5}$, Xavier de Lamballerie ${ }^{6}$, Louis Lambrechts ${ }^{3,8}$ \& \\ Anna-Bella Failloux $x^{1,8} \llbracket$
}

In most of the world, Dengue virus (DENV) is mainly transmitted by the mosquito Aedes aegypti while in Europe, Aedes albopictus is responsible for human DENV cases since 2010. Identifying mutations that make DENV more competent for transmission by Ae. albopictus will help to predict emergence of epidemic strains. Ten serial passages in vivo in Ae. albopictus led to select DENV-1 strains with greater infectivity for this vector in vivo and in cultured mosquito cells. These changes were mediated by multiple adaptive mutations in the virus genome, including a mutation at position 10,418 in the DENV 3'UTR within an RNA stem-loop structure involved in subgenomic flavivirus RNA production. Using reverse genetics, we showed that the 10,418 mutation alone does not confer a detectable increase in transmission efficiency in vivo. These results reveal the complex adaptive landscape of DENV transmission by mosquitoes and emphasize the role of epistasis in shaping evolutionary trajectories of DENV variants.

Vector-borne diseases represent almost one fourth of all emerging infectious diseases worldwide \{Christian 2017\}. Among the emerging diseases, arboviruses occupy the top stair with several million human cases reported annually $^{1}$. Dengue virus (DENV; Flavivirus, Flaviviridae) is by far the most important arboviral disease, with the number of human dengue infection cases exceeding 300 million annually. Clinical manifestations range from mild cases of dengue fever to severe cases of dengue hemorrhagic fever and/or dengue shock syndrome; 96 million are symptomatic dengue fever/hemorrhagic fever leading to an estimated 22,000 human deaths ${ }^{2}$. DENV is comprised of four antigenically distinct but genetically related serotypes referred to as DENV1-4 ${ }^{3}$. All four DENV serotypes are now circulating in Asia, Africa and America ${ }^{4}$. In past centuries, dengue was not an uncommon disease in Europe: the last record of a dengue outbreak in the twentieth century was in Athens, Greece, in 1927-19285. This outbreak was unusual by the number of cases ( 1 million) and the importance of severe clinical symptoms (e.g. hemorrhagic manifestations) leading to deaths $(\sim 1000)$.

After this Greek episode, dengue disappeared from Europe ${ }^{6}$ as the mosquito Aedes aegypti disappeared from Eastern Mediterranean after 1935 through improving sanitation and mosquito control measures 7 . No local transmission of DENV has been reported in Europe until 2010, when clusters of autochthonous cases were reported in Southern France ${ }^{8}$ and Croatia ${ }^{9}$. In France, several transmission episodes were successively reported: 2013-2015 $5^{10-12}, 2018-2019^{13,14}$. The vector was Aedes albopictus, first detected in Europe in 1979 in Albania ${ }^{15}$, then in 1990 in Italy ${ }^{16}$, and today, established in more than 20 European countries ${ }^{17}$. Both species share the same trophic preference for human blood, Ae. aegypti being a strict human-biting mosquito and Ae. albopictus, a mammal-feeding generalist. Ae. aegypti occupies preferentially urban environments while Ae. albopictus colonizes vegetated and rural habitats. These features distinguish them in the transmission of arboviruses in human-mosquito cycles $^{18}$. It is largely admitted that Ae. albopictus plays a minor role compared to Ae. aegypti in DENV transmission ${ }^{19}$. However, unexpectedly, Ae. albopictus from France was shown to be more competent to

\footnotetext{
${ }^{1}$ Arboviruses and Insect Vectors Unit, Institut Pasteur, Paris, France. ${ }^{2}$ Sorbonne Université, Collège doctoral, 75005 Paris, France. ${ }^{3}$ Insect-Virus Interactions Unit, Institut Pasteur, UMR2000, CNRS, Paris, France. ${ }^{4}$ Cluster of Microbial Ecology, Groningen Institute for Evolutionary Life Sciences, University of Groningen, Groningen, The Netherlands. ${ }^{5}$ Laboratory of Virology, Wageningen University, Wageningen, The Netherlands. ${ }^{6}$ Unité des Virus Émergents (UVE: Aix-Marseille Univ-IRD 190-Inserm 1207-IHU Méditerranée Infection), Marseille, France. ${ }^{7}$ Functional Genetics of Infectious Diseases Unit, Institut Pasteur, Paris, France. ${ }^{8}$ These authors contributed equally: Rachel Bellone, Sebastian Lequime, Henri Jupille, Louis Lambrechts and Anna-Bella Failloux. ${ }^{\square}$ email: anna-bella.failloux@pasteur.fr
} 
experimentally transmit DENV-1 strains compared to its counterpart Ae. aegypti from the French West Indies ${ }^{20}$. Contrary to Ae. aegypti, the mosquito Ae. albopictus which is native to South-East Asia, has a broader range of hosts $^{21}$. When Ae. aegypti is absent, Ae. albopictus can be responsible for DENV epidemics, as shown for the outbreaks in the Seychelles islands ${ }^{22}$, Japan ${ }^{23}$, La Réunion Island ${ }^{24}$, and Hawaii ${ }^{25}$. However, to date, Ae. albopictus is considered a minor vector of DENV relative to Ae. aegypti $i^{19}$.

The four DENV serotypes have emerged from sylvatic cycles facilitated by a switch of mosquito vectors from enzootic arboreal mosquitoes to domestic and human-biting Aedes mosquitoes transmitting DENV well-adapted to new mosquito and/or human hosts ${ }^{26}$. As most arboviruses, DENV is capable of rapidly adapting to changes in their environment (or novel hosts) due to the accumulation of one or more specific mutations in the viral genome. For DENV, mutations in the 3' untranslated region (UTR) have previously been linked to increased epidemiological fitness of the virus via a mechanism involving increased expression of 3'UTR-derived subgenomic flavivirus RNA (sfRNA) expression ${ }^{27}$. SfRNA is a known determinant for mosquito transmission of multiple flaviviruses like DENV, Zika virus and West Nile virus ${ }^{28-32}$. Nucleotide substitutions in the 3'UTR reducing or ablating sfRNA expression negatively impact viral infection and transmission rates, suggesting that there is evolutionary pressure on conservation of RNA structures that dictate sfRNA expression in mosquitoes ${ }^{33,34}$. These studies indicate that subtle changes in the viral nucleotide composition can enhance the viral epidemic potential. On the same line, CHIKV has acquired the ability to spread globally owing to a single Ae. albopictus-adaptive mutation E1-226V ${ }^{35}$. This mutation increased the infectivity of CHIKV in Ae. albopictus ${ }^{36,37}$.

We hypothesize that DENV can be selected for enhanced transmission by European Ae. albopictus, which would provide insight into future epidemic DENV strains that could pose a threat to human health. We conducted an experimental evolution study to identify nucleotide changes in the DENV genome by serially passaging DENV-1 isolates from Thailand (30A) and France (1806) in an Ae. albopictus population from Nice, France. Ten total passages were completed after which viral isolates were deep sequenced to identify newly acquired mutations. Importantly, we investigated whether the adaptation to the mosquito vector resulted in enhanced transmission potential or replication rate in mosquitoes. These results exemplify the potential of virus-adaptation studies for the identification of DENV strains likely to emerge.

\section{Results}

European Ae. albopictus are differentially susceptible to DENV-1. Arboviral transmission requires competent mosquitoes. To test whether European populations of Ae. albopictus can sustain local transmission of DENV-1, as reported in France ${ }^{8}$ and Croatia ${ }^{9}$, Ae. albopictus mosquitoes from Alessandria and Genoa (Italy), Cornelia and Martorell (Spain), Nice and Saint-Raphael (France) were experimentally infected with DENV-1 1806 from France or with DENV-1 30A from Thailand. Only engorged females were kept for analysis (samples size indicated in Fig. 1). When examining viral infection rate (Fig. 1a) and dissemination efficiency (Fig. 1b) at 14 and 21 days post-infection (dpi), percentages increased along with the dpi for the majority of virus/mosquito combinations. Within a mosquito population, the viral strain did not play a major role in either infection rate or dissemination efficiency (Fisher's exact test: $p>0.05$ after Bonferroni correction; Supplementary Table S1). In contrast, we observed significant differences between mosquito populations (Fisher's exact test: $p<0.05$; Supplementary Table S2) meaning that the geographic origin of the mosquito population is a critical factor that determines the outcome of viral infection and dissemination. Viral loads in heads (indicative of a successful dissemination from the midgut) did not mostly differ among mosquitoes having disseminated the virus (Fig. 1c, Supplementary Table S3) (Wilcoxon Rank-Sum test: $p>0.05$ ). No viral particles were detected in mosquito saliva meaning no viral transmission (data not shown). To run experimental selection of DENV-1 to Ae. albopictus, we chose mosquitoes from Nice which were collected in the site of the first local cases of dengue in Europe, and exhibited low infection rates and transmission efficiencies (i.e. suggesting a high potential to improve phenotypic effects of adaptation).

Experimental adaptation of DENV-1 to Ae. albopictus. To examine whether DENV-1 can adapt to Ae. albopictus, DENV-1 1806 and DENV-1 30A were passaged 10 times in duplicate in Ae. albopictus mosquitoes from Nice, France, via oral infections followed by viral amplifications of collected saliva on C6/36 cells (Fig. 2). Viral titers of cell culture supernatants collected at each passage fluctuated slightly from passages 1 to 10 (Supplementary Fig. S1). Additionally, the viruses were passaged 10 times in duplicate in Ae. albopictus C6/36 cells as a cell culture control. Full viral genomes were examined by deep sequencing at each passage (1-10) for the two replicates (R1 and R2) of in vivo mosquito infections and for passages 0 (parental strain), 1, 5 and 10 for the C6/36 cell culture control.

The two parental DENV-1 strains, 1806 and 30A, yielded a mean sequencing depth of 68687X (1806) and $133941 \mathrm{X}$ (30A), covering $99.97 \%$ and $100 \%$ of the reference genome at $>100 \mathrm{X}$. With the exception of $30 \mathrm{~A}$ (passage 4, R2 in mosquitoes; covering only $34.62 \%$ of the reference genome at $>100 \mathrm{X}$ ), all passages had a mean sequencing depth between 995X and 211830X, paving between 100 and $99.25 \%$ of the reference genome at $>100 \mathrm{X}$ (Supplementary Fig. S2).

No major changes in single nucleotide variants (SNV) frequencies were detected when DENV-1 isolate was serially passaged on C6/36 cells (Fig. 3a). Remarkably, we did not detect a single mutation that reached consensus level (frequency $>50 \%$ ) in the C6/36 control passages. In contrast, when DENV-1 1806 or 30A was passaged in Ae. albopictus mosquitoes, consensus level variants were detected as soon as passages 2 (DENV-1 1806) and 5 (DENV-1 30A), and a total of 30 consensus level variants were detected at passage 10 (Fig. 3a, Supplementary Table S4). In total, twenty consensus level SNVs were detected in DENV-1 30A (positions 448, 694, 1611, 1768, 1959, 2002, 2200, 2716, 2977, 3442, 5822, 6658, 6728, 7267, 7952, 8149, 8485, 9504, 10,208, 10,258) and 10 in DENV-1 1806 (positions 1840, 2719, 3001, 3757, 4552, 4606, 5667, 7360, 9067, 10,418). Out of the 30 consensus 

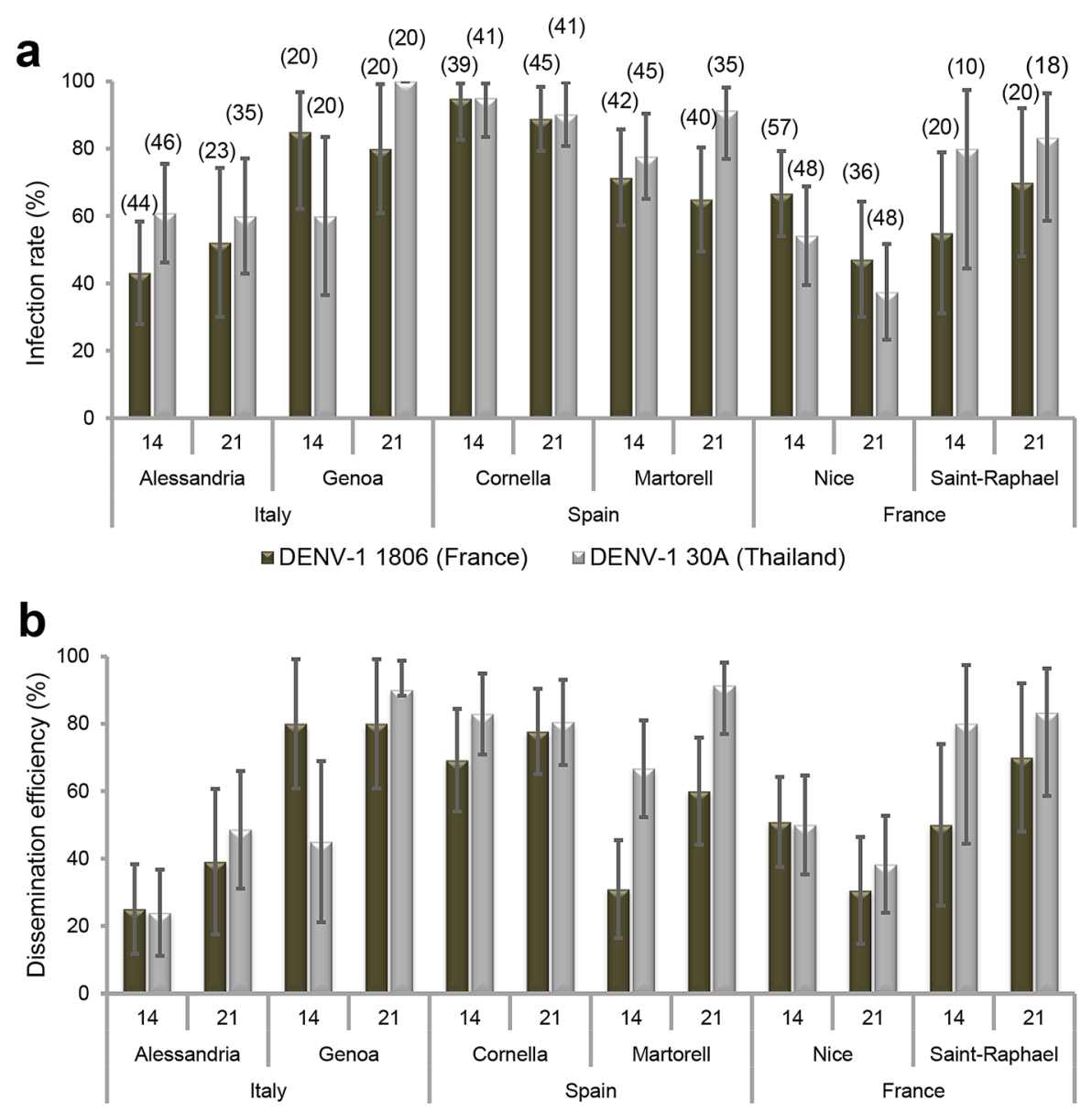

口DENV-1 1806 (France) $\square$ DENV-1 30A (Thailand)

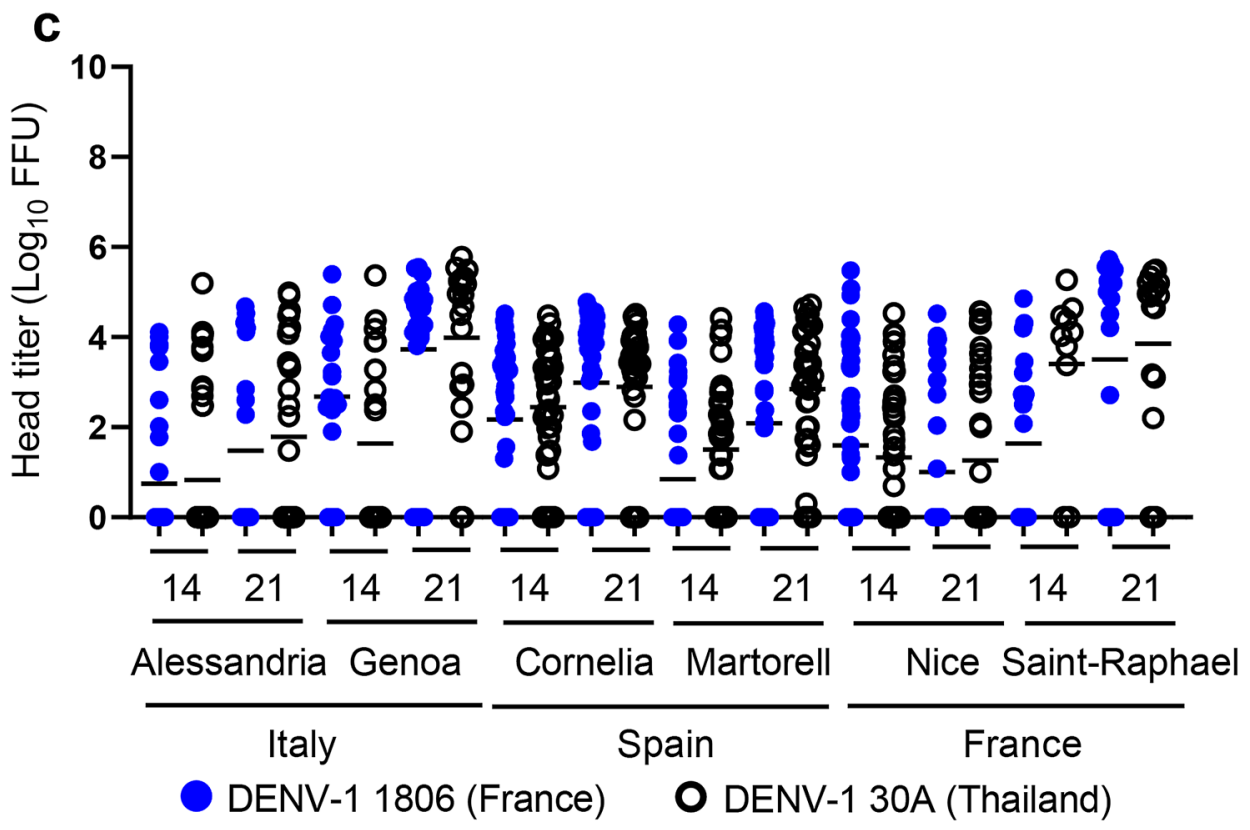

Figure 1. Susceptibilities of six European Ae. albopictus populations to DENV-1 (1806 and 30A): (a) infection rate, (b) dissemination efficiency and (c) viral titers in heads. Adult female mosquitoes were challenged with DENV-1 from France (1806) and Thailand (30A) at a titer of $10^{7} \mathrm{FFU} / \mathrm{mL}$. At 14 and $21 \mathrm{dpi}$, mosquitoes were sacrificed and decapitated. Bodies and heads were homogenized and titrated on C6/36 cells. Infection rates were determined using positive/negative scoring (i.e. without estimating the number of viral particles), while viral titers at sites of dissemination were quantified via focus-forming assay. The error bars correspond to the confidence intervals (95\%) (a,b), and the bar to the mean (c). In brackets is indicated the sample size. 


\section{DENV-1}

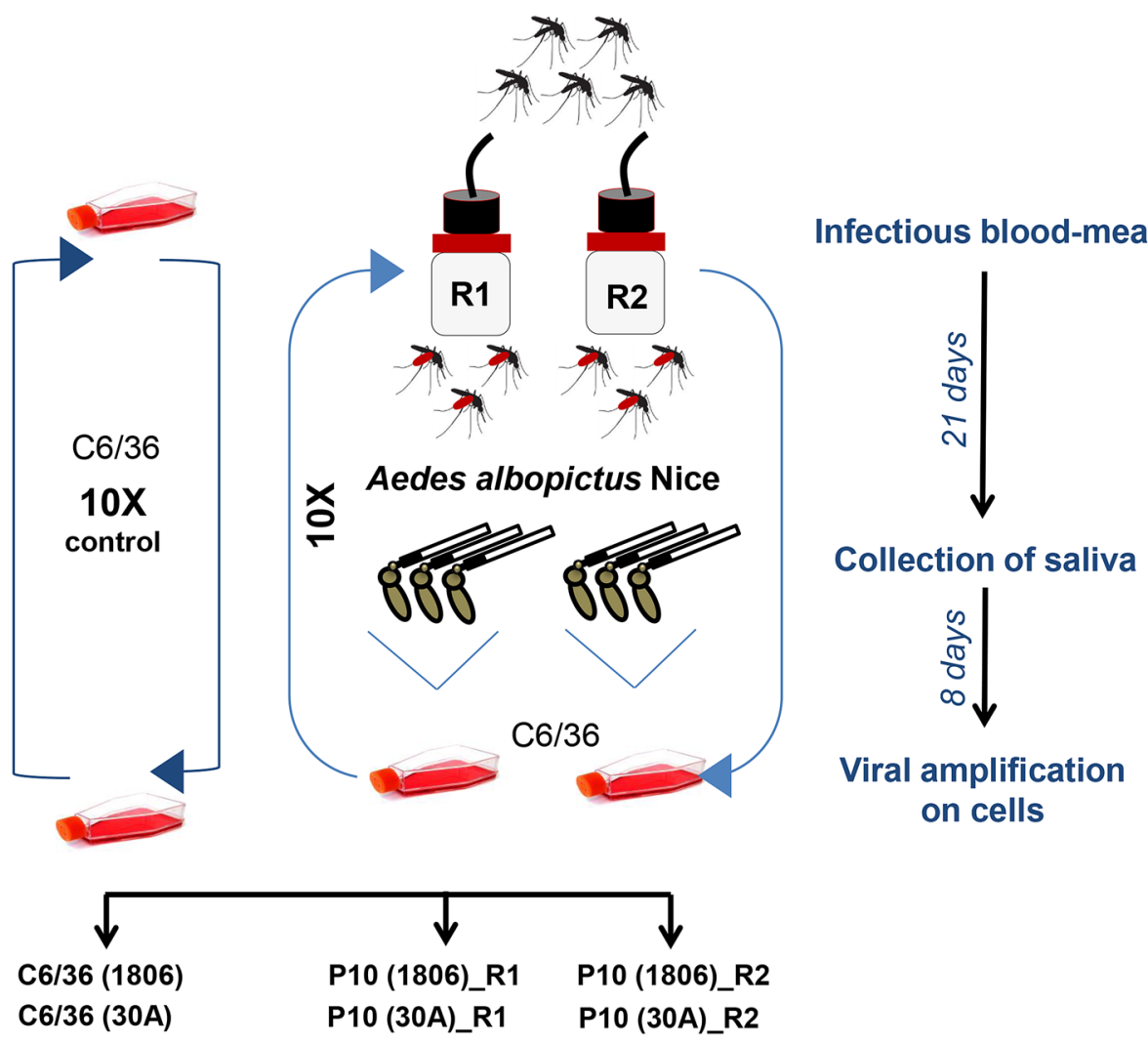

\section{Parental 1806 Parental 30A \\ (France) \\ (Thailand)}

Figure 2. Experimental design for DENV-1 adaptation to Ae. albopictus. The parental strains 1806 (France) and $30 \mathrm{~A}$ (Thailand) were passaged 10 times on a single Ae. albopictus population from Nice, France. Each passage includes: mosquito infectious blood-meal with DENV-1, collection of mosquito saliva at day 21 post-infection, viral amplification of saliva on Ae. albopictus cell cultures for 8 days, and initiation of the next passage using the viral suspension obtained. Control isolates were serially passaged 10 times on C6/36 cells. Two replicates R1 and R2 were performed.

variants, 23 synonymous changes, 6 non-synonymous and one variant located in the 3'UTR were detected (Fig. 3b). The variant located at position 10,418 in the 3'UTR was the only SNV shared between the replicates R1 and R2 of DENV-1 1806. Its frequency increased over the passages, reaching consensus level at passage 4 for replicate 1 and passage 8 for replicate 2 (Fig. 3a). The variant became almost fixed (frequency $>99 \%$ ) at passage 5 for replicate 1 and passage 10 in replicate 2. No SNV was common to the two replicates for DENV-1 30A. These results indicate that DENV-1 accumulates mutations during passaging in Ae. albopictus that likely facilitate virus replication in the mosquito or virus dissemination into the saliva to facilitate transmission.

Ae. albopictus adapted DENV-1 1806 has an increased transmission rate in Ae. albopictus. To investigate whether the mosquito adapted DENV-1 1806 has increased transmission potential as compared to the parental isolate, Ae. aegypti Pazar (Turkey) and Ae. albopictus Nice (France) were provided with an infectious blood-meal containing 107 FFU/mL DENV-1 1806 parental, or replicate of the mosquito passaged virus (R1 and R2). Viral infection rates were high (>40\%) and higher for the P10 viruses in Ae. albopictus at 21 dpi (Fig. 4a,b). Viral dissemination was lower at early dpi but remained high at $21 \mathrm{dpi}$ in both mosquito species with a higher dissemination of $\mathrm{P} 10$ viruses compared to the parental virus (Fig. 4c,d). Transmission was surprisingly low in $A e$. aegypti (Fig. 4e) compared to Ae. albopictus (Fig. 4f) suggesting a stronger effect of salivary glands as a barrier to virus release in saliva of Ae. aegypti. At $21 \mathrm{dpi}$, the two P10 viruses had higher infection rates (Fig. 4b), higher dissemination rates (Fig. $4 \mathrm{~d})$ and higher transmission rates ( 2.5 fold) in Ae. albopictus than their parental strain (Fisher's exact test: $p<10^{-4}$; IR, $p=0.0001 ; \mathrm{DE}, p=0.0001$; TE, $p=0.022$ ). Collectively, these results indicate that the accumulation of adaptive mutations during passaging in Ae. albopictus for the P10_R1 and P10_R2 viruses is beneficial for virus infection, dissemination and thus transmission by Ae. albopictus mosquitoes.

Ae. albopictus adapted DENV-1 1806 has a replicative advantage in RNAi-competent and -deficient mosquito cells. To determine whether the adaptive mutations in DENV-1 1806 after serial pas- 
a

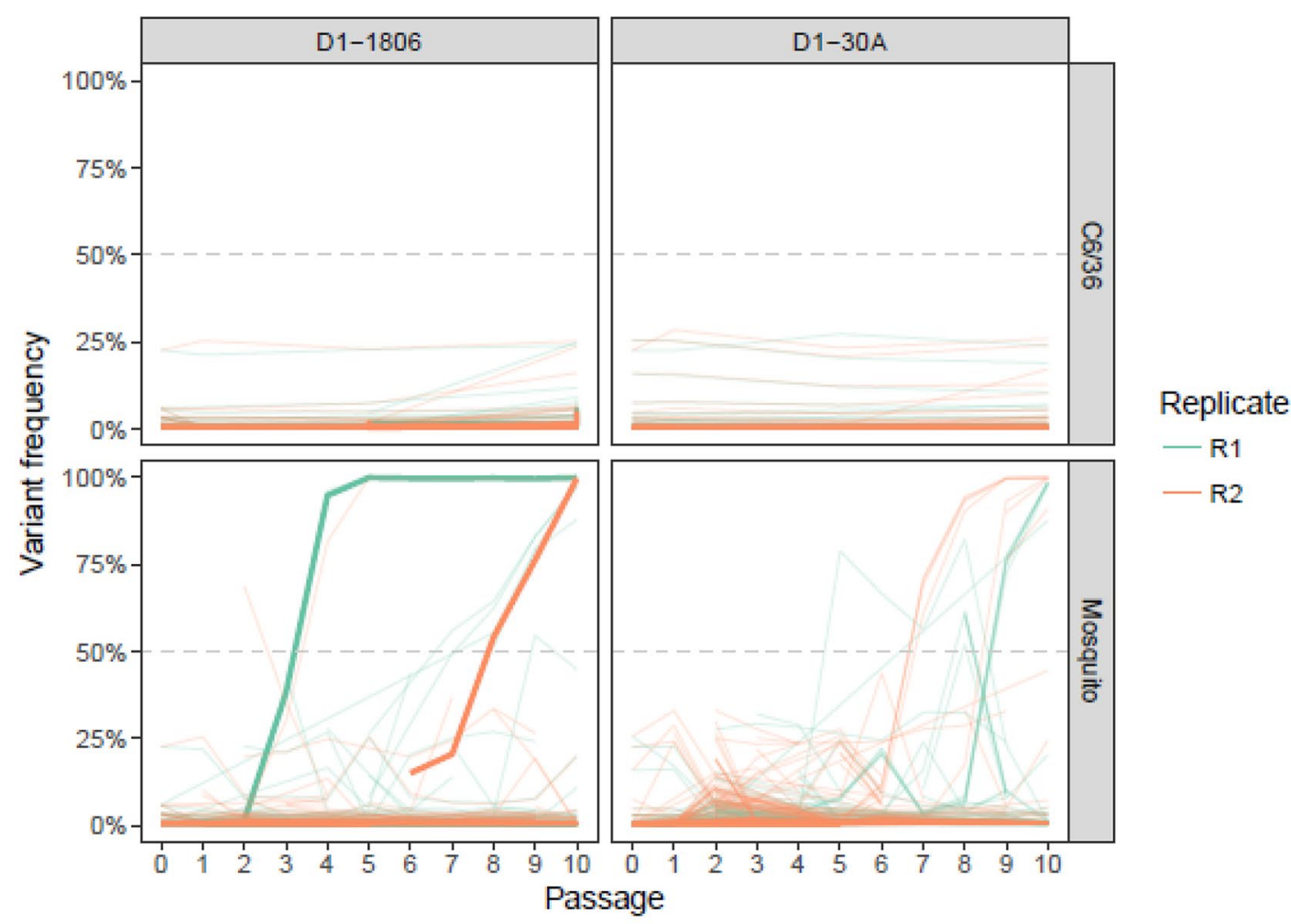

b

\section{D1-1806}

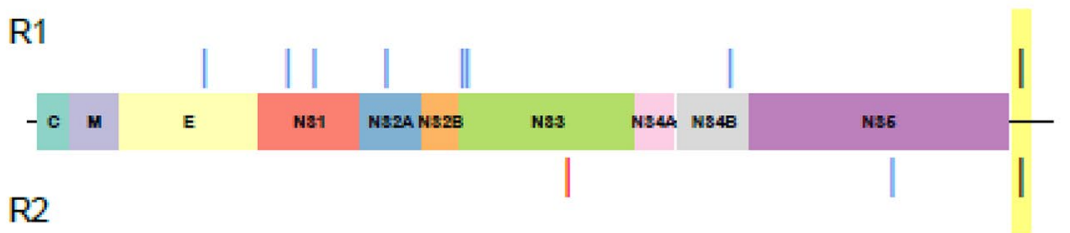

\section{D1-30A}

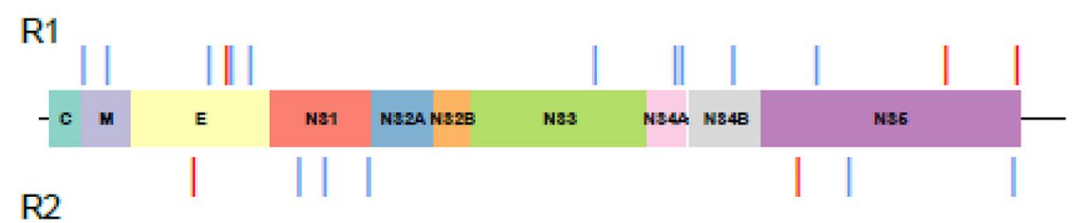

Figure 3. Frequency variation and genomic position of consensus level-reaching variants during passages. (a) The top panels correspond to DENV-1 passaged on C6/36 cells as controls and the bottom panels to DENV-1 passaged on Ae. albopictus mosquitoes. Only mutations with a frequency higher than $50 \%$ were considered. Bold lines represent the variant 10,418 in the 3'UTR in R1 and R2. (b) Variants are represented with a colored segment according to the mutation type (non-coding: black; non-synonymous: red; synonymous: blue). The position of the only shared variant between two replicates is highlighted in yellow.

saging in Ae. albopictus mosquitoes were causing a replicative advantage, we examined the replication kinetics of DENV-1 1806 parental, R1 and R2 in Ae. albopictus C6/36 (RNAi-deficient) and U4.4 (RNAi-competent) cells (Fig. 5a,b) compared to kinetics in HFF cells (Fig. 5c). In C6/36 cells, the parental virus reached slightly lower titers at 6, 24 and $48 \mathrm{~h}$ post-infection (hpi) as compared to the mosquito passaged R1/R2 viruses (Fig. 5a). The mosquito adapted R1/R2 viruses presented a significant increase in viral titer at 24 hpi (R1 (mean \pm SD): $\log _{10}$ $\left.4.42 \pm 0.10 ; \mathrm{R} 2: \log _{10} 4.68 \pm 0.14\right)$ compared to the parental strain $\left(\log _{10} 3.31 \pm 0.15\right)\left(\chi^{2}\right.$ test: $\left.p=0.027\right)$. In U4.4 cells, the same trend was observed (Fig. $5 b)$ with a lower titer at 24 hpi for the parental strain $\left(\log _{10} 2.84 \pm 0.06\right)$ 


\section{Aedes aegypti PAZAR}

\section{Aedes albopictus NICE}

a

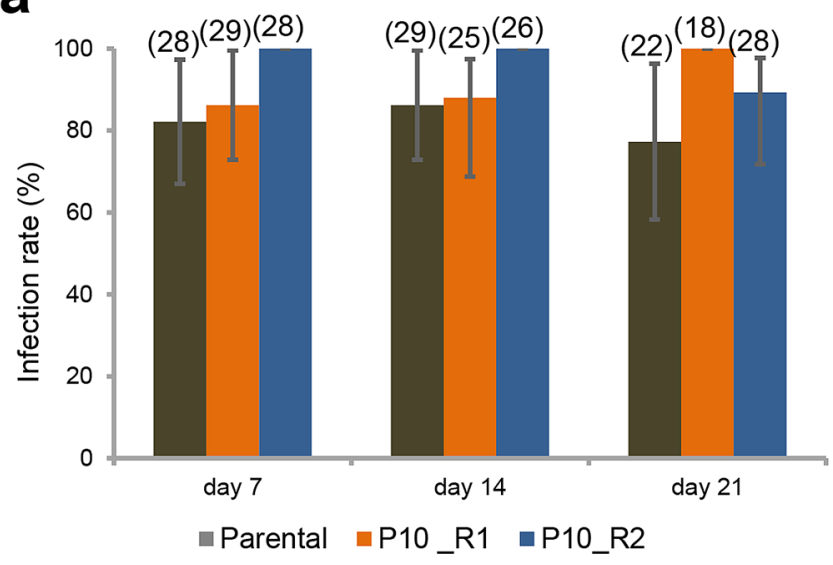

C

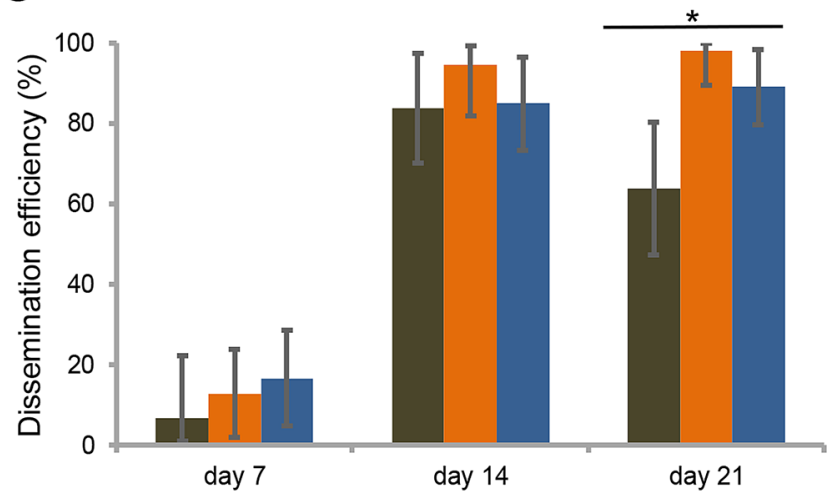

- Parental $\square$ P10_R1 $\square$ P10_R2

e

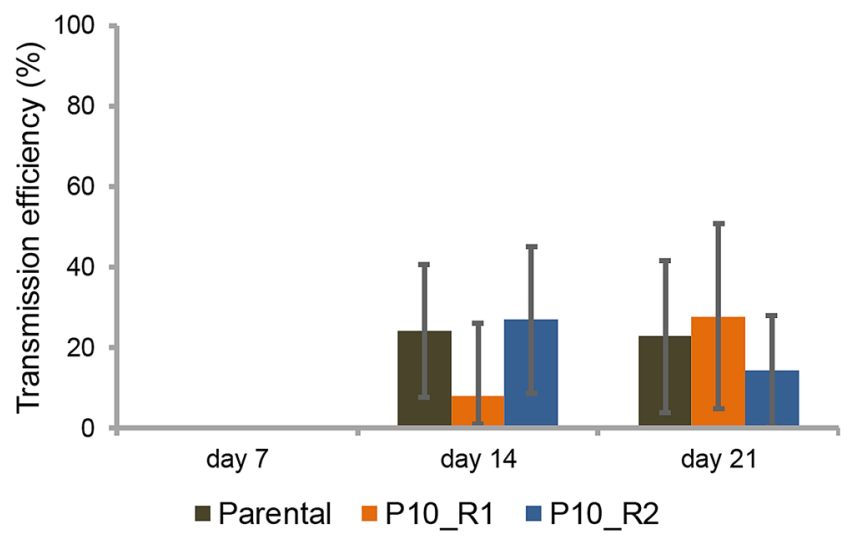

b

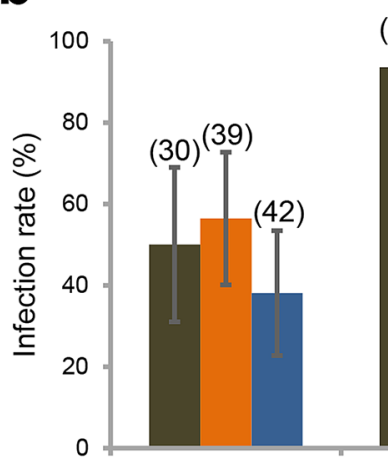

day 7

aParental $\square$ P10_R1 @P10_R2

d

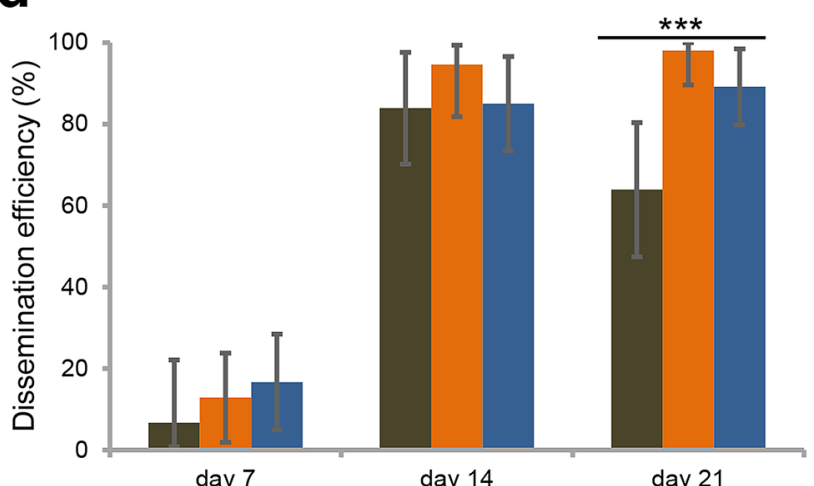

- Parental $\square$ P10_R1 aP10_R2

f

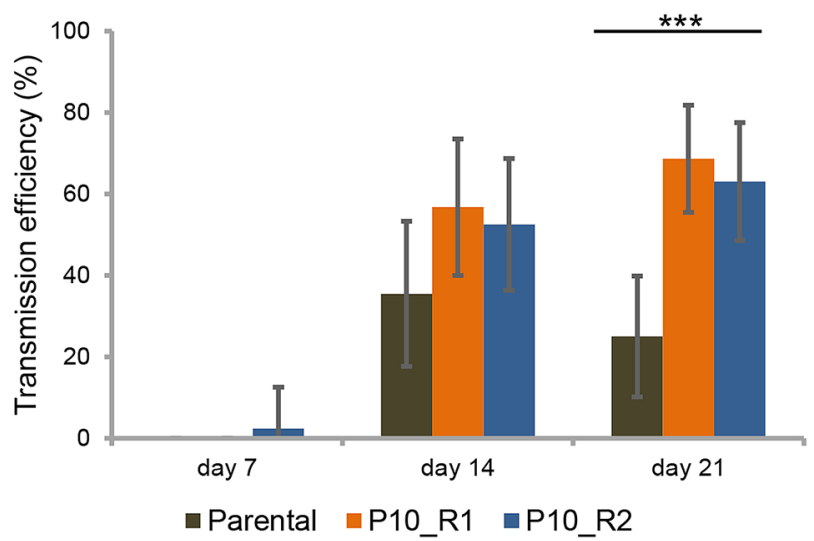

Figure 4. Infection, Dissemination and Transmission of DENV-1 (Parental, P10_R1, and P10_R2) by Ae. aegypti Pazar and Ae. albopictus Nice. Mosquitoes were exposed to blood meals at a titer of $10^{7} \mathrm{FFU} / \mathrm{mL}$. Females were examined at 7,14 and $21 \mathrm{dpi}$. Mosquito body (thorax and abdomen) and head were processed individually to determine (a,b) the infection rate (IR, proportion of mosquitoes with infected body among the engorged mosquitoes) and (c,d) the dissemination efficiency (DE, proportion of mosquitoes with infected head among tested mosquitoes). (e,f) Saliva was collected from individual females to determine the transmission efficiency (TE, proportion of mosquitoes with infectious saliva among tested mosquitoes). The Parental strain corresponds to DENV-1 1806, and P10_R1 and P10_R2 refer, respectively, to replicate 1 and replicate 2 of the 10th in vivo passages of DENV-1 1806 on Ae. albopictus. Asterisks refer to a significant difference $\left({ }^{\star *} p<10^{-3}\right)$. In brackets, the number of mosquitoes tested. The error bars correspond to the confidence intervals (95\%). 


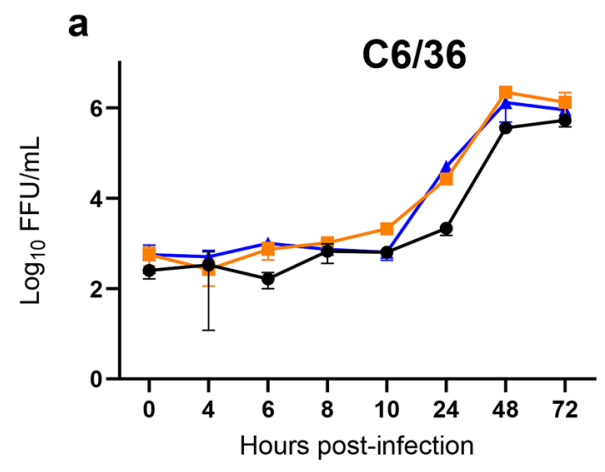

$$
\begin{aligned}
& \text { - Parental } \\
& - \text { P10_R1 } \\
& \text { - P10_R2 }
\end{aligned}
$$

b
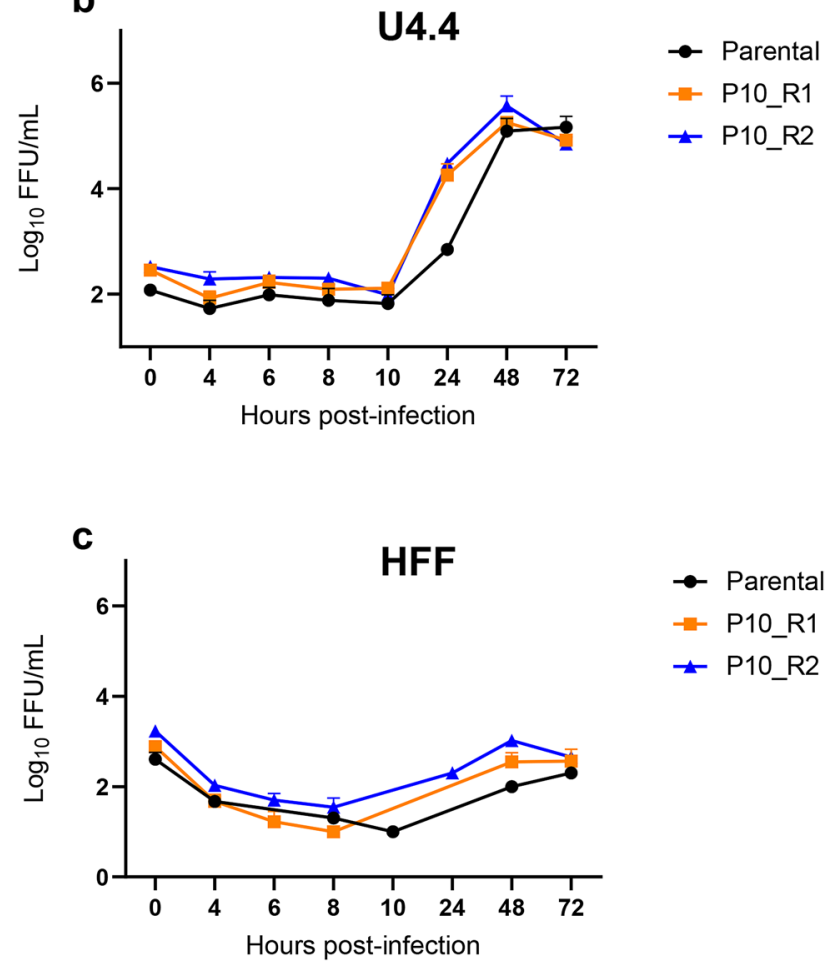

Figure 5. Growth curves of the two passages 10 of DENV-1 1806 strain in three cell lines, (a) Ae. albopictus C6/36 cells, (b) Ae. albopictus U4.4 cells, and (c) human foreskin fibroblasts HFF cells. Cells were infected with the parental strain and the two replicates of the 10th passages of DENV-1 1806 (P10_R1 and P10_R2) at a MOI of 0.1 . Supernatants were collected at $4,6,8,10,24,48$ and $72 \mathrm{~h}$ post-inoculation. The number of infectious viral particles was determined by focus fluorescent assay on Ae. albopictus C6/36 cells. Three replicates were performed for each cell-virus pairing. Error bars show standard deviations.

as opposed to the two P10 viruses (R1: $\log _{10} 4.19 \pm 0.29$; R2: $\log _{10} 4.47 \pm 0.03$ ). In human cells (Fig. $5 \mathrm{c}$ ), viral titers remained between 2 and $3 \log _{10}$ from 0 to $72 \mathrm{hpi}$. These results indicate that the adaptive mutations after serial passaging of DENV-1 1806 in Ae. albopictus mosquitoes increase the replication rate in mosquito cells at $24 \mathrm{hpi}$, that is then recovered at $48 \mathrm{hpi}$.

Vizualisation of substitutions in 3'UTR of DENV-1 1806 on RNA stem-loop structures. The highly structured flavivirus $3^{\prime} \mathrm{UTR}$ is important for virus replication, genome translation and production of non-coding sfRNA ${ }^{38}$. sfRNA is formed as a result of incomplete degradation of the viral genomic RNA by the $5^{\prime}-3^{\prime}$ exoribonuclease XRN1, which stalls on stem loop (SL) and dumbbell (DB) RNA structures in the 3'UTR ${ }^{38,39}$. It has been shown that passaging DENV on mosquito cells can result in high mutation rates in the $3^{\prime} \mathrm{UTR}$ and might alter the abundance of sfRNA during infection ${ }^{33,40}$. The largest sfRNA species, sfRNA1, determines pathogenicity ${ }^{41}$, inhibits host innate immunity ${ }^{27,42}$ and is essential for efficient transmission of flaviviruses by mosquitoes ${ }^{28-32,43,44}$. We therefore investigated if the consensus level mutation 10,418 that occurred in the $3^{\prime} \mathrm{UTR}$ after passaging could lead to changes in the 3'UTR secondary RNA structures and subsequent sfRNA formation. Mutations with an SNV frequency $\geq 0.05$ only occurred in the SL-II and 3'SL structures (Fig. 6a; red nucleotides). When examining mutations in the $3^{\prime} \mathrm{SL}$, the same SNVs were found in the two parental strains and the passages 
10 indicating that those mutations were already present in the initial viral populations and were not selected consequently to serial passages in mosquitoes (Fig. 6b). We observed that the mosquito passaged DENV-1 1806 presented a $\mathrm{U} \rightarrow \mathrm{C}$ substitution at position 10,418 on the top of SL-II, which was not observed for DENV-1 30A. SL-II is the XRN1 stalling structure required for sfRNA2 formation, which requires the presence of a RNA pseudoknot interaction and a complex tertiary folding ${ }^{45}$ (Fig. 6b). Pseudoknot formation and other known tertiary RNA interactions are not expected to be directly disrupted due to the 10,418 sequence change (Fig. 6b). However, the $\mathrm{U} \rightarrow \mathrm{C}$ mutation may indirectly affect the 3D folding of the stem loop. Passages on C6/36 cells also gave rise to lower-frequency mutations in SL-II (frequency<0.2), but none of them reached consensus level.

Increased transmission potential of Ae. albopictus-adapted DENV-1 is not associated with significantly increased sfRNA production. As sfRNAs are generated due to stalling of XRN1 on RNA secondary structures in the viral $3^{\prime} \mathrm{UTR}^{41}$, sequence changes in the $3^{\prime} \mathrm{UTR}$, in particular those that occur in RNA structures involved in XRN1 stalling, may affect the length and expression level of sfRNAs. To investigate whether the production of sfRNA is affected by the mutation 10,418 in DENV-1 1806 R1/R2, a Northern blot analysis was performed using a 3'UTR specific probe on total RNA extracted from U4.4 cells infected with DENV-1 1806 parental, R1 or R2 (Fig. 7). Viral gRNA and abundant sfRNA1 were produced by both the parental and R1/R2 viruses (Fig. 7a). The quantity of sfRNA1 was visually similar across all samples on the gel, although ImageJ quantification of the band intensities revealed that the ratio of sfRNA/gRNA was $\sim 1.5$ fold higher in R1 and R2 samples as compared to the parental samples (Fig. 7b). Minimal amounts of smaller sfRNA species (i.e. sfRNA2, 3, 4) were observed, indicating that DENV-1 predominantly produces sfRNA1 during infection of mosquito cells. These results show that the increased transmission potential of the Ae. albopictus adapted DENV-1 1806 is unlikely to be caused by differences in sfRNA production.

The 10,418 mutation alone does not significantly enhance DENV-1 transmission by Ae. albopictus. To test whether the $\mathrm{U} \rightarrow \mathrm{C}$ substitution at position 10,418 alone would recapitulate the observed phenotype (i.e., increased transmission rate) in Ae. albopictus, three reverse genetic constructs (Parental construct, P10 construct 1 and P10 construct 2) were produced using the ISA method and then sequenced. As expected, the two P10 constructs presented the 10,418 mutation at a frequency close to $100 \%$ (Supplementary Table S5). The genetic constructs also displayed other SNVs at frequencies higher than $5 \%$ but none of them reached consensus level with the exception of one SNV with 52.5\% frequency in P10 construct 1 (5173/nsp3) and two SNVs close to fixation in P10 construct 2 (7321/nsp4b and 9571/nsp5).

Twenty-one days after an infectious blood meal containing the reverse genetic constructs, Ae. albopictus Nice mosquitoes were examined for transmission by collecting mosquito saliva followed by titration on cells. When estimating the transmission efficiency, no significant differences were detected when comparing the five viral strains, the reverse genetic constructs in reference to the template (Parental, P10) and the two P10 constructs ( 1 and 2) (Fisher's exact test: $p>0.05$ ) (Fig. 8a). Similarly, when examining the number of viral particles in individual mosquito saliva, no statistical significance was found whatever the comparison (Wilcoxon Rank-Sum test: $p>0.05$ ) (Fig. $8 b$ ).

To confirm the profile of $\mathrm{P} 10$ construct 1 , we performed a replicate using the same experimental design. TE (Fig. 8c) and the viral load in saliva (Fig. 8d) were determined. We found that the replicate 2 shares the same profile than the replicate 1 (Fig. 8a,b). Altogether, these results indicate that the mutation 10,418 alone does not enhance transmission of DENV-1 in Ae. albopictus.

\section{Discussion}

Our results show that we have successfully adapted DENV-1 to Ae. albopictus, through selection of adaptive mutations including the 10,418 mutation in the $3^{\prime} \mathrm{UTR}$ of the viral genome by sequential passaging in vivo.

Ae. albopictus usually acts as a secondary vector of $\mathrm{DENV}^{21}$, but in the absence of Ae. aegypti, it can act as the main vector in some regions including Europe ${ }^{8-12}$. First detected in Albania in $1979^{15}$, Ae. albopictus is now present in more than 20 European countries ${ }^{17}$. We showed that Ae. albopictus from France, Italy and Spain were susceptible to infection by DENV-1 (Fig. 1), indicating that Ae. albopictus can act as a vector for DENV in Europe. The main sources of introductions in Europe were mosquitoes from Italy, which were previously imported from North America ${ }^{46}$. Recurrent introduction events have contributed to increase the genetic diversity of European Ae. albopictus populations ${ }^{47}$, an important factor shaping vector competence ${ }^{19}$.

Here, we experimentally selected DENV-1 isolates for enhanced transmission by Ae. albopictus. Our experimental procedure was designed to accelerate the selection process of DENV-1 by serial passages in Ae. albopictus mosquitoes without alternation in the mammalian host. After 10 passages in Ae. albopictus collected in Nice, France, we successfully adapted DENV-1 1806 and DENV-1 30A to Ae. albopictus through the accumulation of adaptive mutations across the genome, although only a single mutation was fixed in both replicates for the 1806 isolate. Importantly, for DENV-1 1806, these adaptive mutations increased the infection, dissemination and transmission rates of DENV-1 by Ae. albopictus (Fig. 4). Furthermore, growth kinetics of the DENV-1 1806 viruses were increased in both RNAi-competent U4.4 and RNAi-deficient C6/36 cells, indicating that the mutations cause an increase in viral replicative fitness in cell cultures regardless of a functional RNAi machinery (Fig. 5). These mosquito-selected viral variants were less adapted to replicate on mammalian cells ${ }^{48}$. In a similar approach, Stapleford et al. ${ }^{49}$ succeeded in monitoring the selection of epidemic variants of CHIKV adapted to Ae. albopictus consolidating the idea that in vivo approaches can contribute in predicting new variants able to emerge and displace currently circulating viral strains.

We identified a mutation located at position 10,418 in the highly structured 3'UTR of the DENV-1 genome. Specifically, the mutation was present in the SL-II RNA structure (Fig. 6). This region is required for the 


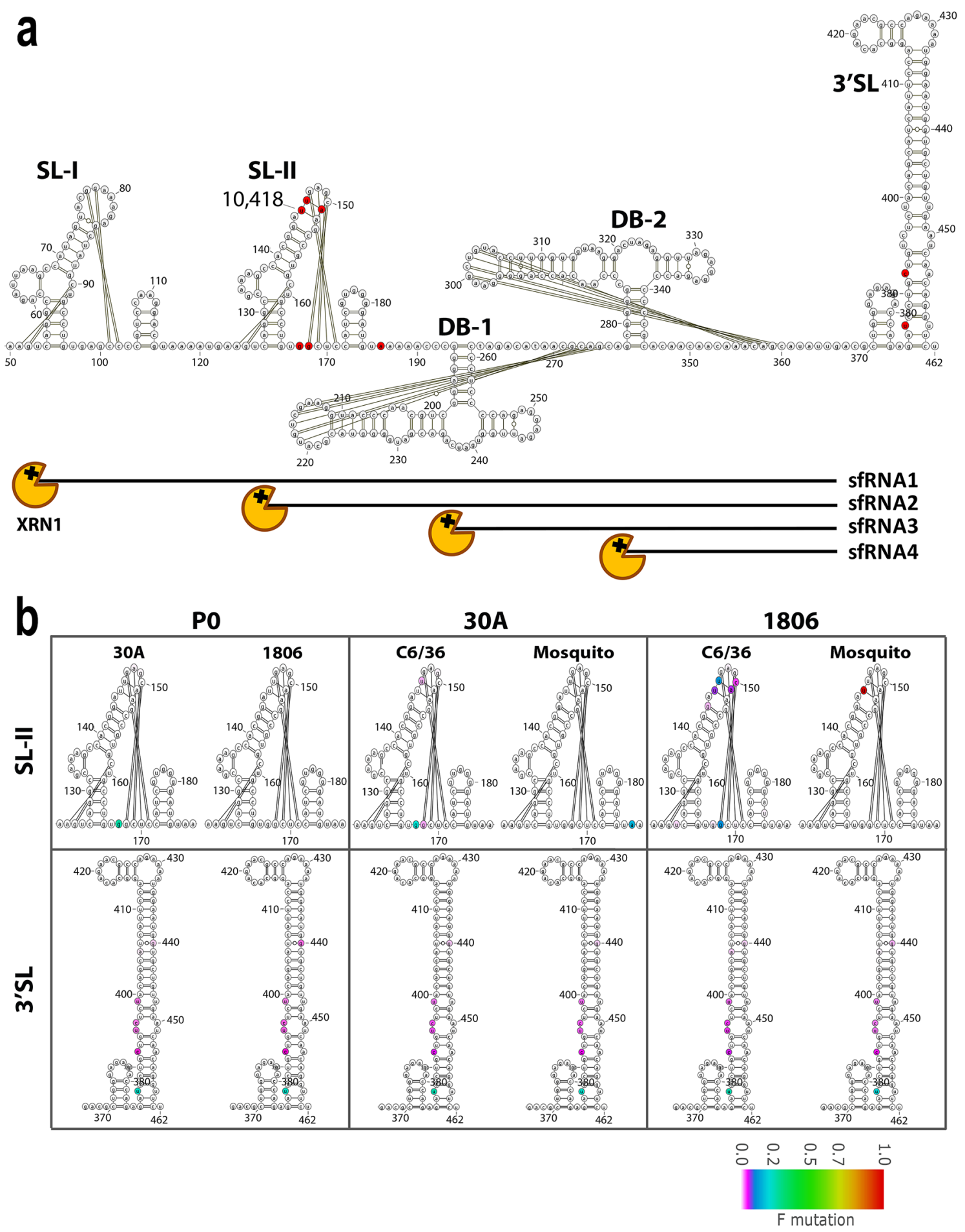

Figure 6. Analysis of mutation frequencies in the DENV-1 3'UTR. (a) Schematic overview of the DENV-1 3'UTR secondary RNA structure, indicating from $5^{\prime}$ to $3^{\prime}$ the stem loop (SL)-I, SL-II, dumbbell (DB)-1, DB-2 and 3'SL RNA structures. Single nucleotide variants with a frequency $\geq 0.05$ after 10 passages of DENV-1 1806 or $30 \mathrm{~A}$ in either $\mathrm{C} 6 / 36$ or mosquitoes are highlighted in red. Pseudoknots and other tertiary RNA interactions are indicated by the black lines. (b) Analysis of the mutation frequencies in SL-II and the 3'SL of the parental DENV-1 30A and 1806 sequences, and passages P10 (1806) and P10 (30A). The mutation frequency is indicated by color on a scale from 0 (white) to 1 (red). The figure was prepared using the free software VARNA RNA editing package version 3-93. (https://varna.lri.fr/index.php?lang=en\&page=downloads\&css=varna) and modified with Adobe Illustrator CC. 
a
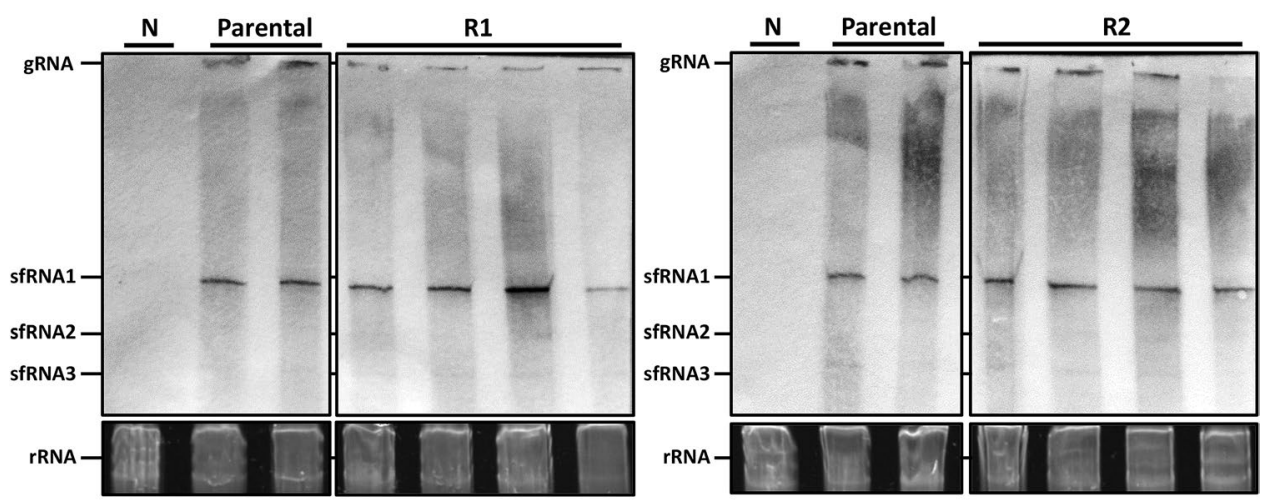

b

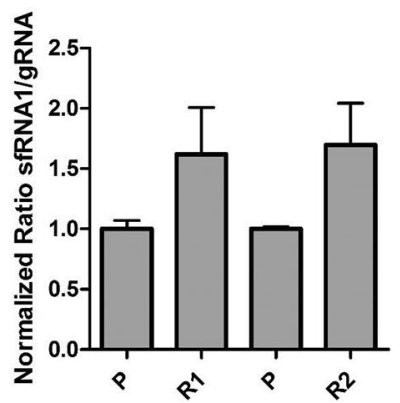

Figure 7. Northern blot detection of sfRNA production after C6/36 cells infection with DENV-1 1806. (a) Visualization of sfRNA. $5 \mu \mathrm{g}$ of total RNA from the parental and the two replicates P10_R1 and P10_R2 of DENV-1 1806 or non-infected cells (N) was size separated on a 6\% Polyacrylamide/Urea gel. One gel was run for the R1 samples (left) and one for the R2 samples (right), including the negative and parental samples on each gel. Then, RNAs were blotted onto Hybond-N paper and subjected to northern-blotting with a DENV-1 3'UTR specific probe. The northern blot panels have been cropped from the same original blot, which is available in the supplemental material (Supplementary Fig. S3.). The bands shown correspond to the DENV genomic RNA (gRNA) and subgenomic flavivirus RNA (sfRNA). As loading control, the ribosomal RNA (rRNA) from the EtBr stained gel are shown. (b) Quantification of the ratio of sfRNA to gRNA production. Band intensities were determined using the 'Measure' function in ImageJ. The intensity of the background (lane N) was subtracted from the readings before the ratio sfRNA/gRNA was calculated by dividing the intensity of the sfRNA by the intensity of the gRNA band for each sample, and then normalized to the average ratio of the parental samples. The statistics were performed using a two-tailed unpaired t-test.

production of sfRNA2 ${ }^{41}$. XRN1 stalls at SL and dumbbell (DB) RNA structures within the $3^{\prime} \mathrm{UTR}$, which results in accumulating sfRNAs of different sizes ${ }^{41,50}$. The stalling of XRN1 occurs due to steric hindrance caused by interactions of pseudoknots (PK) and other tertiary RNA structures ${ }^{45,51}$. Prediction of RNA structures involved in XRN1 stalling (the so-called xrRNAs) with Mfold has proven to be an useful starting point but undeniably has limitations, e.g. pseudoknots cannot be predicted and 3D RNA folding is not taken into account. Although Mfold predictions and visual pseudoknot mapping have helped to elucidate mechanisms of XRN1 stalling ${ }^{41,45,50}$, the exact structural basis for XRN1 stalling, the involvement of a unique three-way junction and internal tertiary interactions were only revealed by determining the crystal structure of several xrRNAs ${ }^{52,53}$.

In mammalian cells, sfRNA is essential for inducing pathogenicity ${ }^{41}$, and acts as an antagonist of innate immune responses ${ }^{27,42}$. In mosquito cells, sfRNA has been reported as an antagonist of the RNAi response in vitro ${ }^{54,55}$ and contributes to enhance the in vivo infection of mosquitoes and further dissemination from the midgut into the haemocoe ${ }^{32}$ and subsequent salivary gland infection ${ }^{44}$. Villordo et al. ${ }^{40}$ previously demonstrated that when passaging DENV-2 20 times in C6/36 mosquito cells, SL-II is highly mutated while the upstream SL-I mutates mostly upon passaging in mammalian cells. The mutations in SL-II were shown to increase DENV-2 replication in mosquito cells ${ }^{40}$. The mutation that we found at the position 10,418 in SL-II is in line with these findings, supporting the mutation pressure on SL-II in vivo, although we did not observe significant mutations during passaging in $\mathrm{C} 6 / 36$ cells.

For DENV-2, it has been shown that during replication in human cells, mainly sfRNA1 is produced, while mosquito-adapted DENV-2 accumulates more abundant sfRNA3 and sfRNA4 ${ }^{33}$. We show that DENV-1 1806 produced abundant sfRNA1 while quantities of sfRNA2,3 and possibly sfRNA4 were below the detection limit (Fig. 7), suggesting possible differences in the production of sfRNA species between DENV-1 and DENV-2. Despite the presence of the 10,418 mutation, the Ae. albopictus adapted DENV-1 1806 did not show a significantly altered production of sfRNA species. Although we cannot exclude an effect on cellular binding partners 
a

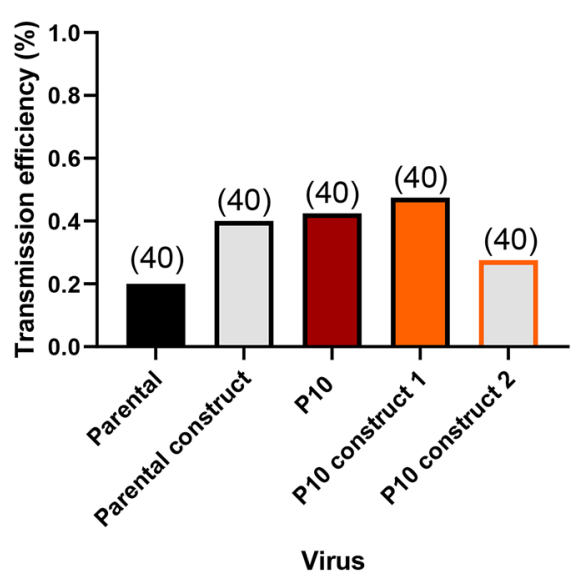

C

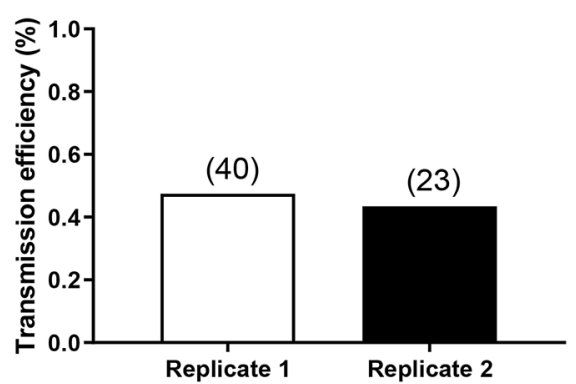

b

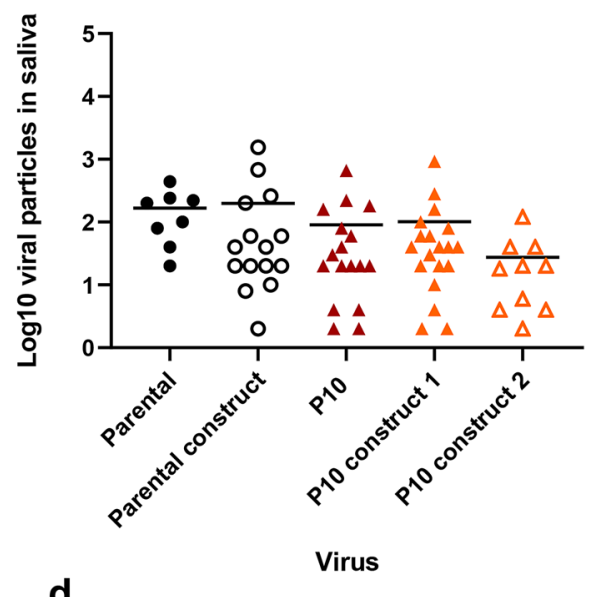

d

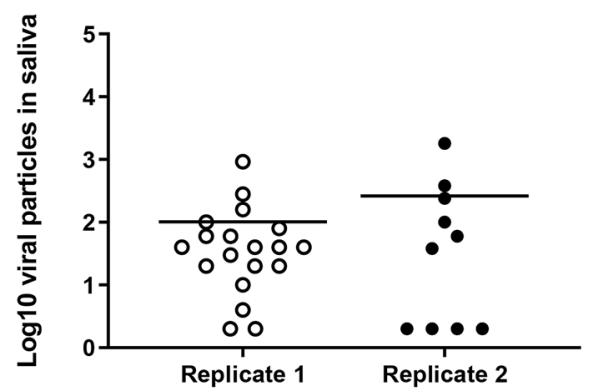

Figure 8. Reverse genetic constructs with the 10,418 mutation do not show higher transmission in Ae. albopictus. (a) Transmission of reverse genetic constructs (Parental construct, P10 construct 1, P10 construct 2) by Ae. albopictus Nice with reference to Parental and P10 strains. Twenty-one days after an infectious blood meal at a titer of $10^{7} \mathrm{FFU} / \mathrm{mL}$, mosquitoes were processed for saliva collection to determine the transmission efficiency (TE, proportion of mosquitoes with infectious saliva among tested mosquitoes). (b) Viral loads in saliva were estimated by focus fluorescent assay on Ae. albopictus C6/36 cells. (c,d) A second replicate using the $\mathrm{P} 10$ construct 1 was performed. Bars indicate the mean.

that might require an intact 3'UTR for their interaction with the viral genome ${ }^{38}$, it is unlikely that sfRNAs were a primary driver of DENV-1 adaptation to Ae. albopictus.

We used reverse genetics to evaluate the effect of the 10,418 mutation on DENV-1 transmission by Ae. albopictus mosquitoes in vivo, but our results did not provide experimental support for a phenotypic effect of the 10,418 mutation alone. For two different genetic constructs harboring the 10,418 mutation (together with different adventitious mutations), there was no detectable difference in transmission efficiency. Introducing the 10,418 did not recapitulate the adapted phenotype of the P10 viruses and points to a more complex adaptive landscape than a single-mutation effect. Because our genetic constructs focused on the 10,418 mutation did not include other mutations present in the P10 viruses, it implies that the enhanced transmission phenotype reflected the combined effect of several mutations. Such epistatic relationships have been documented to shape the adaptive landscapes of $\mathrm{CHIKV}^{56,57}$ and more recently $\mathrm{DENV}^{58}$. Interestingly, the 10,418 mutation was the only shared mutation among replicates of adapted viruses (Fig. 3), indicating that DENV-1 adaptation to Ae. albopictus can result from distinct evolutionary trajectories involving different sets of mutations.

Our experimental approach has succeeded in enhancing the transmission of DENV-1 by multiple passages in the Ae. albopictus vector. This may ultimately lead to new insights into the mechanisms of arbovirus transmission by mosquitoes.

\section{Materials and methods}

Cell cultures. Ae. albopictus $\mathrm{C} 6 / 36$ cells were maintained at $28^{\circ} \mathrm{C}$ in Leibovitz L-15 medium supplemented with non-essential amino-acids (NEAA) (1X), 10\% fetal bovine serum (FBS), 100 units/mL penicillin and $100 \mu \mathrm{g} / \mathrm{mL}$ streptomycin. These cells are defective in typical siRNAs, the hallmark of exogenous RNAi mediated antiviral immunity ${ }^{59}$; they are highly permissive to viral replication. Ae. albopictus U4.4 cells were maintained in L-15 medium supplemented with non-essential amino-acids (1X), 10\% FBS, 100 units/mL penicillin and $100 \mu \mathrm{g} / \mathrm{mL}$ streptomycin at $28^{\circ} \mathrm{C}$. HFF (Human Foreskin Fibroblast; kindly provided by T. Couderc, Institut Pasteur) cells were maintained at $37^{\circ} \mathrm{C}, 5 \% \mathrm{CO}_{2}$ in Dulbecco's Modified Eagle medium (DMEM) supplemented with pyruvate, $10 \% \mathrm{FBS}, 100$ units $/ \mathrm{mL}$ penicillin and $100 \mu \mathrm{g} / \mathrm{mL}$ streptomycin. The human embryonic kidney HEK-293 cells (ATCC number CCL-1573) were grown at $37^{\circ} \mathrm{C}$ with $5 \% \mathrm{CO}_{2}$ in tissue-culture flasks with vented 


\begin{tabular}{|l|l|l|l|l|}
\hline Species & Mosquito population & Country & Date of collection & Generation used for mosquito infections \\
\hline \multirow{4}{*}{ Aedes albopictus } & Nice & France & August 2011 & F3, F11 \\
\cline { 2 - 5 } & Saint-Raphaël & France & November 2012 & F3 \\
\cline { 2 - 5 } & Alessandria & Italy & November 2012 & F1 \\
\cline { 2 - 5 } & Genoa & Italy & October 2012 & F2 \\
\cline { 2 - 5 } & Cornella & Spain & July 2012 & F2 \\
\cline { 2 - 5 } & Martorell & Spain & July 2012 & F2 \\
\hline Aedes aegypti & Pazar & Turkey & 2016 & F4 \\
\hline
\end{tabular}

Table 1.. Details on mosquito populations used for experimental infections with DENV-1.

caps, in a minimal essential medium (MEM, Life Technologies) supplemented with 7\% FBS, 1\% PenicillinStreptomycin and $1 \mathrm{X}$ NEAA.

Viruses. We used two DENV-1 strains isolated from DF cases: DENV-1 1806 (genotype V) from an autochthonous case from Nice, France in 2010 (provided by the National Reference Center of Arboviruses, France) and DENV-1 30A (genotype I) from a patient in Kamphaeng Phet, Thailand in 2010 (provided by the Afrims, Thailand and under accession number HG316482 in GenBank). The 2nd passage of DENV-1 1806 on African green monkey kidney Vero cells ${ }^{60}$ and the 2 nd passage of DENV-1 30A on C6/36 Ae. albopictus cells ${ }^{61}$ were used for mosquito infections. Serial dilutions were used to determine the titer of viral stocks that was expressed in focus-forming units $(\mathrm{FFU}) / \mathrm{mL}$.

Mosquito strains. Six populations of Ae. albopictus have been established from eggs: Genoa (Italy), Alessandria (Italy), Cornella (Spain), Martorell (Spain), Nice Jean Archet (France), and Saint-Raphael (France) (Table 1). They were tested to appraise vector competence to DENV-1 isolates. Together with Ae. albopictus Nice Jean Archet (France), Ae. aegypti Pazar (Turkey) was utilized to compare vector competence using viruses isolated after 10 passages on Ae. albopictus. Eggs were collected from ovitraps and sent to the Institut Pasteur in Paris, where they were reared in standardized conditions. After hatching, larvae were distributed in pans containing a yeast tablet renewed as needed in $1 \mathrm{~L}$ of tap water. Adults were placed in cages maintained at $28 \pm 1{ }^{\circ} \mathrm{C}$, at relative humidity of $80 \%$ and a light:dark cycle of $16 \mathrm{~h}: 8 \mathrm{~h}$, with free access to $10 \%$ sucrose solution. Oral infection experiments were performed using mosquitoes from the F2-F11 generations. Owing to the limited number of mosquitoes, only one biological replicate was performed for each pairing population-virus.

Mosquito infections. One-week-old females were starved $24 \mathrm{~h}$ prior an infectious blood-meal in a BSL-3 laboratory. Five batches of 60 mosquito females were then allowed to feed for $15 \mathrm{~min}$ through a piece of pork intestine covering the base of a Hemotek feeder containing the infectious blood-meal maintained at $37^{\circ} \mathrm{C}$. Only engorged females were kept and incubated under controlled conditions $\left(28 \pm 1^{\circ} \mathrm{C}\right.$, relative humidity of $80 \%$, light:dark cycle of $16 \mathrm{~h}: 8 \mathrm{~h}$ ).

For vector competence assays. Fourteen and 21 days after an infectious blood-meal provided at a titer of $10^{7}$ FFU/mL, vector competence was assessed based on two phenotypes: (1) viral infection of mosquito and (2) viral dissemination from the midgut into mosquito general cavity. Infection rate (IR) was determined as the proportion of mosquitoes with infected midgut and dissemination efficiency (DE) was defined as the percentage of mosquitoes with virus detected in heads suggesting a successful viral dissemination from the midgut. IR and DE were calculated by titrating body and head homogenates.

For serial passages. As the first autochthonous DENV cases were reported in Nice in $2010^{8}$, Ae. albopictus isolated in Nice was used to achieve the experimental selection of DENV-1 isolates (Fig. 2). Mosquitoes were orally infected with DENV-1 supernatant provided in a blood-meal at a final titer of $10^{6.5} \mathrm{FFU} / \mathrm{mL}$ using the hemotek system. Engorged mosquitoes were incubated at $28^{\circ} \mathrm{C}$ for $19-21$ days and then processed for saliva collection. 15-25 saliva were pooled and the volume of the pool was adjusted to $600 \mu \mathrm{L}$ with DMEM prior to filtration through a Millipore $\mathrm{H}$ membrane $(0.22 \mu \mathrm{m})$. An aliquot of $300 \mu \mathrm{L}$ of each sample was used to inoculate a sub-confluent flask $\left(25 \mathrm{~cm}^{2}\right)$ of $\mathrm{C} 6 / 36$ Ae. albopictus cells. After $1 \mathrm{~h}$, the inoculum was discarded and cells were rinsed once with medium. Five $\mathrm{mL}$ of DMEM medium complemented with $2 \%$ FBS was added and cells were incubated for 8 days at $28^{\circ} \mathrm{C}$. Cell culture supernatants were then collected and provided to mosquitoes to run the next passage. Passages P1 to P3 were performed with mosquitoes of the F3 generation and passages P4 to $\mathrm{P} 10$ with mosquitoes of the $\mathrm{F} 4$ generation. $\mathrm{C} 6 / 36$ supernatants collected at each passage were used undiluted for the next mosquito blood-meal. Ten passages were performed. Control isolates corresponded to serially passaged viruses on C6/36 cells to identify mutations resulting from genetic drift or adaptation to insect cell line; $500 \mu \mathrm{L}$ of the previous passage were used to inoculate the next flask of C6/36 cells. Two biological replicates R1 and R2 were performed to test the variability between samples submitted to the same protocol of selection. Vector competence using the parental and P10 isolates was assessed by calculating: (1) infection rate (IR, proportion of mosquitoes with infected midgut), (2) dissemination efficiency (DE, proportion of mosquitoes able to dis- 
seminate the virus from the midgut among tested mosquitoes), and (3) transmission efficiency (TE, proportion of mosquitoes with the virus detected in saliva among tested mosquitoes).

Virus deep sequencing. Total RNA was extracted from cell culture supernatant using QIAamp Viral RNA Mini Kit (Qiagen, Germany) and DNAse treated (Turbo DNAse, Life Technologies, USA). Following purification with magnetic beads (Agencourt RNAClean XP, Beckman Coulter, California, USA), RNA was reverse transcribed using Transcriptor High Fidelity cDNA Synthesis Kit and a specific 3'-UTR DENV-1 primer (Roche Applied Science, Mannheim, Germany), d1a5B 5'-AGAACCTGTTGATTCAACRGC- $3^{\prime 62}$. Second strand was then synthetized in a unique reaction with E. coli DNA ligase (New England Biolabs, Massachusetts, USA), E. coli DNA polymerase I (New England Biolabs), E. coli RNAse H (New England Biolabs) in second strand synthesis buffer (New England Biolabs). After purification with magnetic beads (Agencourt AMPure XP, Beckman Coulter), dsDNA was quantified with fluorometric method (Quant-iT PicoGreen dsDNA, Invitrogen, Massachusetts, USA).

Sequencing libraries were prepared using Nextera XT DNA Library Preparation Kit (Illumina, San Diego, USA), multiplexed and sequenced in single end in two independent runs on an Illumina NextSeq 500 platform using a mid-output 150-cycle v2 kit (Illumina). Reads were trimmed (Trimmomatic v0.33) ${ }^{63}$ after demultiplexing (bcl2fastq v.2.15.0, Illumina) to remove adaptor sequences, and reads shorter than 32 nucleotides were discarded.

Full-length genome of the DENV-1 1806 was assembled de novo using Ray v2.0.0 ${ }^{64}$ with the original stock sample. The newly assembled DENV genome contig was extended in $3^{\prime}$ and $5^{\prime}$ using closest BLAST hit full DENV-1 genome (accession number EU482591). This chimeric construct was used to map reads used for assembly using Bowtie 2 v2.1.0 $0^{65}$. Alignment file was converted, sorted and indexed using Samtools v0.1.1966. Sequencing depth was assessed using bedtools v2.17.067. Single nucleotide variants and their frequency were called using LoFreq ${ }^{\star}$ v2.1.1 $1^{68}$ and used to correct the chimeric construct. Only nucleotides with $>10 \mathrm{X}$ coverage were conserved for generating the consensus sequence. A final full-length genome sequence for DENV-1 1806 strain was deposited to GenBank (accession number MG518567).

After quality control, reads from all samples were mapped to the newly assembled DENV-1 1806 strain genome sequence or previously sequenced reference genome KDH0030A (accession number HG316482) using Bowtie v2.1.0 $0^{65}$. The alignment file was converted, sorted and indexed using Samtools v0.1.1966, and the sequencing depth was assessed for each sample using bedtools v2.17. ${ }^{67}$. Single nucleotide variants (SNVs) and their frequency were then called using LoFreq ${ }^{*} \mathrm{v} 2.1 .1^{68}$, with the built-in SNV filtration using the default parameters, and their effect at the amino-acid level was assessed by SNPgenie v1.2 ${ }^{69}$.

RNA structure modeling in silico. The Mfold Web server was used with standard settings and flat exterior loop type ${ }^{70}$ to fold the secondary RNA structures, which were then visualized using the VARNA RNA editing package ${ }^{71}$. Pseudoknot RNA interactions were drawn as previously described for DENV ${ }^{45,72}$. Mutation frequencies of individual nucleotides were determined by averaging the nucleotide allele frequency from the deep sequencing results of the duplicates per treatment.

Virus growth curves. To measure viral replicative fitness, growth curves were conducted in Ae. albopictus C6/36 and U4.4 mosquito cells, and Human Foreskin Fibroblasts (HFF) cells. Confluent cell monolayers were prepared and inoculated with viruses simultaneously in triplicates at a MOI of $0.1 \mathrm{PFU} /$ cell. Cells were incubated for $1 \mathrm{~h}$ in appropriate conditions and viral inoculum was removed to eliminate free virus. Five $\mathrm{mL}$ of medium supplemented with $2 \%$ FBS were then added and mosquito cells were incubated at $28^{\circ} \mathrm{C}$ (mosquito cells) or $37^{\circ} \mathrm{C}$ (human cells). At various times $(4,6,8,10,24,48$ and $72 \mathrm{~h}$ ) post-inoculation (pi), supernatants were collected and titrated by focus fluorescent assay on Ae. albopictus C6/36 cells. After incubation at $28^{\circ} \mathrm{C}$ for 5 days, plates were stained using hyper immune ascetic fluid specific to DENV as primary antibody (Millipore, Molsheim, France). A Fluorescein-conjugated goat anti-mouse was used as the second antibody (Thermofisher). Three viral strains were used: the parental strain and two 10th passages, P10_R1 and P10_R2. Viral titer was expressed in FFU/mL. Three biological replicates were performed for each cell-virus pairing.

RNA isolation and Northern blotting. Total RNA was isolated from cell monolayers using TRIzol reagent (Invitrogen, Massachusetts, France) following the manufacturer's protocol. Mosquito DENV-1 infected bodies were homogenized individually in $500 \mu \mathrm{L}$ of Leibovitz L15 medium (Invitrogen) supplemented with $2 \%$ fetal bovine serum for $1 \mathrm{~min}$ at maximum speed. Homogenates were then filtered with a filter unit $(0.22 \mu \mathrm{m})$ (Ultrafree MC-GV, Merck, New Jersey, USA). Two samples of each filtrate were inoculated onto monolayers of Ae. albopictus $\mathrm{C} 6 / 36$ cell culture in 6 -well plates. After incubation at $28^{\circ} \mathrm{C}$ for 6 days, samples were homogenized with $1 \mathrm{~mL}$ TRIzol reagent. RNA isolations were performed using the standard TRIzol protocol. Samples were eluted in $30 \mu \mathrm{L}$ RNase-free Milli-Q water and stored at $-80^{\circ} \mathrm{C}$ until further processing. A DENV-1 $3^{\prime} \mathrm{UTR}$ specific probe was generated by PCR reaction with GoTaq Polymerase (Promega, Wisconsin, USA) containing DIG DNA-labelling mix (Roche) and primers DENV-1 3'UTR FW (AGTCAGGCCAGATTAAGCCATAGTACGG) and DENV-1 3'UTR RV (ATTCCATTTTCTGGCGTTCTGTGCCTGG) using cDNA from cells infected with DENV-1 1806 as a template. Five micrograms of total RNA was subjected to sfRNA-optimized northern blot as has been described previously ${ }^{32}$. Briefly, total RNA was denatured and size separated on $6 \%$ polyacrylamide-7 M urea- $0.5 \times$ Tris-borate-EDTA (TBE) gel for $1.45 \mathrm{~h}$ at $150 \mathrm{~V}$. The RNA was semi-dry-blotted on a Hybond-N membrane, UV cross-linked and pre-hybridized for $1 \mathrm{~h}$ at $50{ }^{\circ} \mathrm{C}$ in modified Church buffer containing $10 \%$ formamide. DENV-1 $3^{\prime}$ UTR specific Dig-labelled probe was denatured and blots were hybridized overnight at $50{ }^{\circ} \mathrm{C}$ in modified church/10\% formamide buffer containing $2 \mu \mathrm{L}$ of DIG-labelled probe. Blots were developed with AP-labeled anti-DIG antibodies and NBT-BCIP solution before observing the signal using a Bio-Rad Gel 
Doc scanner. Quantification of band signal intensities was performed in ImageJ by transforming the image to 8-bit format, inverting the image, and analyzing the band intensity using the measure function. The Ratio sfRNA/gRNA was calculated by dividing the intensity of the sfRNA by the intensity of the gRNA band for each sample, and then normalized to the average ratio of the parental samples.

ISA reverse genetics. The $\mathrm{T}>\mathrm{C}$ mutation at position 10,418 identified at passage 10 was inserted into a DENV-1 1806 backbone using the ISA (Infectious Subgenomic Amplicons) reverse genetics method as previously described ${ }^{73}$.

Preparation of subgenomic DNA fragments. The viral genome was amplified by RT-PCR from the DENV-1 1806 viral RNA as three overlapping DNA fragments. Two additional fragments were de novo synthesized (Genscript) and amplified by PCR (primers are listed in S6 Table). The first primer consisted of the human cytomegalovirus promoter (pCMV) and the second primer of the last 367 nucleotides of the 3'UTR of the DENV-1 1806 with or without the 10,418 $\mathrm{T}>\mathrm{C}$ mutation and the hepatitis delta ribozyme followed by the simian virus 40 polyadenylation signal (HDR/SV40pA) (sequences are listed in Supplementary Text S1). RT mixes were prepared using the superscript IV reverse transcriptase kit (Life Technologies, CA, USA) and PCR mixes using the Q5 High-Fidelity PCR Kit (New England Biolabs, MA, USA) following the manufacturer's instructions. RT were performed in the following conditions: $25^{\circ} \mathrm{C}$ for $10 \mathrm{~min}$ followed by $37^{\circ} \mathrm{C}$ for $50 \mathrm{~min}$ and $70{ }^{\circ} \mathrm{C} 15 \mathrm{~min}$. PCR amplifications were performed in the following conditions: $98^{\circ} \mathrm{C}$ for $30 \mathrm{~s}$ followed by 35 cycles of $98^{\circ} \mathrm{C}$ for $10 \mathrm{~s}, 62^{\circ} \mathrm{C}$ for $30 \mathrm{~s}$, $72{ }^{\circ} \mathrm{C}$ for $2 \mathrm{~min} 30 \mathrm{~s}$, with a $2 \mathrm{~min}$ final elongation at $72^{\circ} \mathrm{C}$. PCR product sizes and quality were controlled by running gel electrophoresis and DNA fragments were purified using a QIAquick PCR Purification Kit (Qiagen, Hilden, Germany).

Cell transfection. HEK-293 cells were seeded into six-well cell culture plates one day prior to transfection. Cells were transfected with $2 \mu \mathrm{g}$ of an equimolar mix of the five DNA fragments using lipofectamine 3000 (Life Technologies) following the manufacturer's instructions. Each transfection was performed in five replicates. After incubating for $24 \mathrm{~h}$, the cell supernatant medium was removed and replaced by fresh cell culture medium. Seven days post-transfection, cell supernatant medium was passaged two times using six-well cell culture plates of confluent C6/36 cells. Cells were subsequently inoculated with $100 \mu \mathrm{L}$ of diluted (1/3) cell supernatant media, incubated $1 \mathrm{~h}$, washed with PBS $1 \mathrm{X}$, and incubated 7 days with $3 \mathrm{~mL}$ of medium. Remaining cell supernatant medium was stored at $-80^{\circ} \mathrm{C}$. The second passage was used to produce virus stock solutions of DENV-1 1806 WT and mutant viruses.

Transmission efficiency was assessed 21 days after an infectious blood meal containing the Parental, the Parental construct, the P10 strain, the P10 constructs (1 and 2) provided separately at a titer of $10^{7} \mathrm{FFU} / \mathrm{mL}$.

Statistical analyses. Statistical analyses were conducted using the STATA software (StataCorp LP, Texas, and USA). $p$ values $>0.05$ were considered non-significant. If necessary, the significance level of each test was adjusted based on the number of tests run, according to the sequential method of Bonferroni ${ }^{74}$.

Ethics statement. The Institut Pasteur animal facility has received accreditation from the French Ministry of Agriculture to perform experiments on live animals in compliance with the French and European regulations on care and protection of laboratory animals (EC Directive 2010/63, French Law 2013-118, February 6th, 2013). This study was approved by the Ethics Committee \#89 (animal experimentation ethics committee of the Institut Pasteur) and registered under the reference APAFIS\#6573-2016061412077987 v2. Mice were only used for mosquito rearing as a blood source, according to approved protocol.

\section{Data availability}

The data that support the findings of this study are all in the manuscript.

Received: 26 August 2020; Accepted: 6 October 2020

Published online: 27 October 2020

\section{References}

1. Liang, G., Gao, X. \& Gould, E. A. Factors responsible for the emergence of arboviruses; strategies, challenges and limitations for their control. Emerg. Microbes Infect. 4, e18. https://doi.org/10.1038/emi.2015.18 (2015).

2. Bhatt, S. et al. The global distribution and burden of dengue. Nature 496, 504-507. https://doi.org/10.1038/nature12060 (2013).

3. Calisher, C. H. et al. Antigenic relationships between flaviviruses as determined by cross-neutralization tests with polyclonal antisera. J. Gen. Virol. 70(Pt 1), 37-43. https://doi.org/10.1099/0022-1317-70-1-37 (1989).

4. Guzman, M. G. \& Harris, E. Dengue. Lancet 385, 453-465. https://doi.org/10.1016/S0140-6736(14)60572-9 (2015).

5. Anon. The dengue epidemic in Greece. Leag. Nations Mon. Epidemiol. Rep. 7, 334 (1928).

6. Rezza, G. Dengue and chikungunya: long-distance spread and outbreaks in naive areas. Pathog. Glob. Health 108, 349-355. https ://doi.org/10.1179/2047773214Y.0000000163 (2014).

7. Curtin, T. J. Status of Aedes aegypti in the Eastern Mediterranean. J. Med. Entomol. 4, 48-50 (1967).

8. La Ruche, G. et al. First two autochthonous dengue virus infections in metropolitan France, September 2010. Euro Surveillance Bulletin Europeen sur les Maladies Transmissibles = European Communicable Disease Bulletin 15, 19676 (2010).

9. Gjenero-Margan, I. et al. Autochthonous dengue fever in Croatia, August-September 2010. Euro Surveillance: Bulletin Europeen sur les Maladies Transmissibles = European Communicable Disease Bulletin 16, 19805 (2011).

10. Marchand, E. et al. Autochthonous case of dengue in France, October 2013. Euro Surveillance: Bulletin Europeen sur les Maladies Transmissibles = European Communicable Disease Bulletin 18, 20661 (2013). 
11. Giron, S. et al. Nouvelles apparitions de cas autochtones de dengue en région Provence-Alpes-Côte d'Azur, France, août-septembre 2014. Bull. Epidémiol. Hebd. 13-14, 217-225 (2015).

12. Succo, T. et al. Autochthonous dengue outbreak in Nimes, South of France, July to September 2015. Euro Surveillance: Bulletin Europeen sur les Maladies Transmissibles = European Communicable Disease Bulletin 21, 5-11. https://doi.org/10.2807/1560-7917. ES.2016.21.21.30240 (2016).

13. Franke, F. et al. Émergences de dengue et de chikungunya en France métropolitaine, 2010-2018. Bull. Epidémiol. Hebd. 19-20, 374-382 (2019).

14. Santé Publique France. Chikungunya, dengue et zika - Données de la surveillance renforcée en France métropolitaine en 2020. (2020). https://www.santepubliquefrance.fr/maladies-et-traumatismes/maladies-a-transmission-vectorielle/chikungunya/artic les/donnees-en-france-metropolitaine/chikungunya-dengue-et-zika-donnees-de-la-surveillance-renforcee-en-france-metropolit aine-en-2019.

15. Adhami, J. \& Reiter, P. Introduction and establishment of Aedes (Stegomyia) albopictus skuse (Diptera: Culicidae) in Albania. J. Am. Mosq. Control Assoc. 14, 340-343 (1998).

16. Sabatini, A., Raineri, V., Trovato, G. \& Coluzzi, M. Aedes albopictus in Italy and possible diffusion of the species into the Mediterranean area. Parassitologia 32, 301-304 (1990).

17. Medlock, J. M. et al. An entomological review of invasive mosquitoes in Europe. Bull. Entomol. Res. 105, 637-663. https://doi. org/10.1017/S0007485315000103 (2015).

18. Lounibos, L. P. \& Kramer, L. D. Invasiveness of Aedes aegypti and Aedes albopictus and vectorial capacity for Chikungunya Virus. J. Infect. Dis. 214, S453-S458. https://doi.org/10.1093/infdis/jiw285 (2016).

19. Lambrechts, L., Scott, T. W. \& Gubler, D. J. Consequences of the expanding global distribution of Aedes albopictus for dengue virus transmission. PLoS Negl. Trop. Dis. 4, e646. https://doi.org/10.1371/journal.pntd.0000646 (2010).

20. Vega-Rua, A. et al. High efficiency of temperate Aedes albopictus to transmit chikungunya and dengue viruses in the Southeast of France. PLoS ONE 8, e59716. https://doi.org/10.1371/journal.pone.0059716 (2013).

21. Paupy, C., Delatte, H., Bagny, L., Corbel, V. \& Fontenille, D. Aedes albopictus, an arbovirus vector: from the darkness to the light. Microbes Infect. Institut Pasteur 11, 1177-1185. https://doi.org/10.1016/j.micinf.2009.05.005 (2009).

22. Metselaar, D. et al. An outbreak of type 2 dengue fever in the Seychelles, probably transmitted by Aedes albopictus (Skuse). Bull. World Health Organ. 58, 937-943 (1980).

23. Sabin, A. B. Research on dengue during World War II. Am. J. Trop. Med. Hyg. 1, 30-50 (1952).

24. Coulanges, P., Clercy, Y., Jousset, F. X., Rodhain, F. \& Hannoun, C. Dengue at Reunion: isolation of a strain at the Pasteur Institute of Madagascar. Bull. Soc. Pathol. Exot. 72, 205-209 (1979).

25. Effler, P. V. et al. Dengue fever, Hawaii, 2001-2002. Emerg. Infect. Dis. 11, 742-749. https://doi.org/10.3201/eid1105.041063 (2005).

26. Wang, E. et al. Evolutionary relationships of endemic/epidemic and sylvatic dengue viruses. J. Virol. 74, 3227-3234 (2000).

27. Manokaran, G. et al. Dengue subgenomic RNA binds TRIM 25 to inhibit interferon expression for epidemiological fitness. Science (New York, N.Y.) 350, 217-221. https://doi.org/10.1126/science.aab3369 (2015).

28. Slonchak, A. et al. Zika virus noncoding RNA suppresses apoptosis and is required for virus transmission by mosquitoes. Nat. Commun. 11, 2205. https://doi.org/10.1038/s41467-020-16086-y (2020).

29. Goertz, G. P. et al. Subgenomic flavivirus RNA binds the mosquito DEAD/H-box helicase ME31B and determines Zika virus transmission by Aedes aegypti. Proc. Natl. Acad. Sci. USA 116, 19136-19144. https://doi.org/10.1073/pnas.1905617116 (2019).

30. Yeh, S. C. \& Pompon, J. Flaviviruses produce a subgenomic flaviviral RNA that enhances mosquito transmission. DNA Cell Biol. 37, 154-159. https://doi.org/10.1089/dna.2017.4059 (2018).

31. Pompon, J. et al. Dengue subgenomic flaviviral RNA disrupts immunity in mosquito salivary glands to increase virus transmission. PLoS Pathog. 13, e1006535. https://doi.org/10.1371/journal.ppat.1006535 (2017).

32. Goertz, G. P. et al. Noncoding subgenomic flavivirus RNA is processed by the mosquito RNA interference machinery and determines West Nile virus transmission by Culex pipiens Mosquitoes. J. Virol. 90, 10145-10159. https://doi.org/10.1128/JVI.00930-16 (2016).

33. Filomatori, C. V. et al. Dengue virus genomic variation associated with mosquito adaptation defines the pattern of viral non-coding RNAs and fitness in human cells. PLoS Pathog. 13, e1006265. https://doi.org/10.1371/journal.ppat.1006265 (2017).

34. Pallares, H. et al. Zika virus sfRNA generation requires cooperativity between duplicated RNA structures that are essential for productive infection in human cells. J. Virol. https://doi.org/10.1128/JVI.00343-20 (2020).

35. Schuffenecker, I. et al. Genome microevolution of chikungunya viruses causing the Indian Ocean outbreak. PLoS Med. 3, e263. https://doi.org/10.1371/journal.pmed.0030263 (2006).

36. Tsetsarkin, K. A., Vanlandingham, D. L., McGee, C. E. \& Higgs, S. A single mutation in chikungunya virus affects vector specificity and epidemic potential. PLoS Pathog. 3, e201. https://doi.org/10.1371/journal.ppat.0030201 (2007).

37. Vazeille, M. et al. Two Chikungunya isolates from the outbreak of La Reunion (Indian Ocean) exhibit different patterns of infection in the mosquito Aedes albopictus. PLoS ONE 2, e1168. https://doi.org/10.1371/journal.pone.0001168 (2007).

38. Roby, J. A., Pijlman, G. P., Wilusz, J. \& Khromykh, A. A. Noncoding subgenomic flavivirus RNA: multiple functions in West Nile virus pathogenesis and modulation of host responses. Viruses 6, 404-427. https://doi.org/10.3390/v6020404 (2014).

39. Goertz, G. P. \& Pijlman, G. P. Dengue non-coding RNA: TRIMmed for transmission. Cell Host Microbe 18, 133-134. https://doi. org/10.1016/j.chom.2015.07.009 (2015).

40. Villordo, S. M., Filomatori, C. V., Sanchez-Vargas, I., Blair, C. D. \& Gamarnik, A. V. Dengue virus RNA structure specialization facilitates host adaptation. PLoS Pathog. 11, e1004604. https://doi.org/10.1371/journal.ppat.1004604 (2015).

41. Pijlman, G. P. et al. A highly structured, nuclease-resistant, noncoding RNA produced by flaviviruses is required for pathogenicity. Cell Host Microbe 4, 579-591. https://doi.org/10.1016/j.chom.2008.10.007 (2008).

42. Schuessler, A. et al. West Nile virus noncoding subgenomic RNA contributes to viral evasion of the type I interferon-mediated antiviral response. J. Virol. 86, 5708-5718. https://doi.org/10.1128/JVI.00207-12 (2012).

43. Vogels, C. B. et al. Winter activity and aboveground hybridization between the two biotypes of the West Nile virus vector Culex pipiens. Vector Borne Zoonotic Dis. 15, 619-626. https://doi.org/10.1089/vbz.2015.1820 (2015).

44. Pompon, J. et al. A Zika virus from America is more efficiently transmitted than an Asian virus by Aedes aegypti mosquitoes from Asia. Sci. Rep. 7, 1215. https://doi.org/10.1038/s41598-017-01282-6 (2017).

45. Chapman, E. G., Moon, S. L., Wilusz, J. \& Kieft, J. S. RNA structures that resist degradation by Xrn1 produce a pathogenic dengue virus RNA. Elife 3, e01892. https://doi.org/10.7554/eLife.01892 (2014).

46. Manni, M. et al. Genetic evidence for a worldwide chaotic dispersion pattern of the arbovirus vector Aedes albopictus. PLoS Negl. Trop. Dis. 11, e0005332. https://doi.org/10.1371/journal.pntd.0005332 (2017).

47. Sherpa, S., Rioux, D., Pougnet-Lagarde, C. \& Despres, L. Genetic diversity and distribution differ between long-established and recently introduced populations in the invasive mosquito Aedes albopictus. Infect. Genet. Evol. 58, 145-156. https://doi. org/10.1016/j.meegid.2017.12.018 (2018).

48. Vasilakis, N. et al. Mosquitoes put the brake on arbovirus evolution: experimental evolution reveals slower mutation accumulation in mosquito than vertebrate cells. PLoS Pathog. 5, e1000467. https://doi.org/10.1371/journal.ppat.1000467 (2009).

49. Stapleford, K. A. et al. Emergence and transmission of arbovirus evolutionary intermediates with epidemic potential. Cell Host Microbe 15, 706-716. https://doi.org/10.1016/j.chom.2014.05.008 (2014). 
50. Funk, A. et al. RNA structures required for production of subgenomic flavivirus RNA. J. Virol. 84, 11407-11417. https://doi. org/10.1128/JVI.01159-10 (2010).

51. Akiyama, B. M., Eiler, D. \& Kieft, J. S. Structured RNAs that evade or confound exonucleases: function follows form. Curr. Opin. Struct. Biol. 36, 40-47. https://doi.org/10.1016/j.sbi.2015.12.006 (2016).

52. Chapman, E. G. et al. The structural basis of pathogenic subgenomic flavivirus RNA (sfRNA) production. Science 344, 307-310. https://doi.org/10.1126/science.1250897 (2014).

53. Akiyama, B. M. et al. Zika virus produces noncoding RNAs using a multi-pseudoknot structure that confounds a cellular exonuclease. Science 354, 1148-1152. https://doi.org/10.1126/science.aah3963 (2016).

54. Moon, S. L. et al. Flavivirus sfRNA suppresses antiviral RNA interference in cultured cells and mosquitoes and directly interacts with the RNAi machinery. Virology 485, 322-329. https://doi.org/10.1016/j.virol.2015.08.009 (2015).

55. Schnettler, E. et al. Noncoding flavivirus RNA displays RNA interference suppressor activity in insect and Mammalian cells. J. Virol. 86, 13486-13500. https://doi.org/10.1128/JVI.01104-12 (2012).

56. Tsetsarkin, K. A. et al. Epistatic roles of E2 glycoprotein mutations in adaption of chikungunya virus to Aedes albopictus and Ae. aegypti mosquitoes. PLoS ONE 4, e6835. https://doi.org/10.1371/journal.pone.0006835 (2009).

57. Tsetsarkin, K. A., Chen, R., Sherman, M. B. \& Weaver, S. C. Chikungunya virus: evolution and genetic determinants of emergence. Curr. Opin. Virol. 1, 310-317. https://doi.org/10.1016/j.coviro.2011.07.004 (2011).

58. Syenina, A. et al. Positive epistasis between viral polymerase and the $3^{\prime}$ untranslated region of its genome reveals the epidemiologic fitness of dengue virus. Proc. Natl. Acad. Sci. USA 117, 11038-11047. https://doi.org/10.1073/pnas.1919287117 (2020).

59. Brackney, D. E. et al. C6/36 Aedes albopictus cells have a dysfunctional antiviral RNA interference response. PLoS Negl. Trop. Dis. 4, e856. https://doi.org/10.1371/journal.pntd.0000856 (2010).

60. Grandadam, M. et al. Chikungunya virus, southeastern France. Emerg. Infect. Dis. 17, 910-913. https://doi.org/10.3201/eid17 05.101873 (2011).

61. Fansiri, T. et al. Genetic mapping of specific interactions between Aedes aegypti mosquitoes and dengue viruses. PLoS Genet. 9, e1003621. https://doi.org/10.1371/journal.pgen.1003621 (2013).

62. Christenbury, J. G. et al. A method for full genome sequencing of all four serotypes of the dengue virus. J. Virol. Methods 169, 202-206. https://doi.org/10.1016/j.jviromet.2010.06.013 (2010).

63. Bolger, A. M., Lohse, M. \& Usadel, B. Trimmomatic: a flexible trimmer for Illumina sequence data. Bioinformatics 30, 2114-2120. https://doi.org/10.1093/bioinformatics/btu170 (2014).

64. Boisvert, S., Raymond, F., Godzaridis, E., Laviolette, F. \& Corbeil, J. Ray Meta: scalable de novo metagenome assembly and profiling. Genome Biol. 13, R122. https://doi.org/10.1186/gb-2012-13-12-r122 (2012).

65. Langmead, B. \& Salzberg, S. L. Fast gapped-read alignment with Bowtie 2. Nat. Methods 9, 357-359. https://doi.org/10.1038/nmeth $1923(2012)$.

66. Li, H. et al. The sequence alignment/map format and SAMtools. Bioinformatics 25, 2078-2079. https://doi.org/10.1093/bioinforma tics/btp352 (2009).

67. Quinlan, A. R. \& Hall, I. M. BEDTools: a flexible suite of utilities for comparing genomic features. Bioinformatics 26, 841-842. https://doi.org/10.1093/bioinformatics/btq033 (2010).

68. Wilm, A. et al. LoFreq: a sequence-quality aware, ultra-sensitive variant caller for uncovering cell-population heterogeneity from high-throughput sequencing datasets. Nucleic Acids Res. 40, 11189-11201. https://doi.org/10.1093/nar/gks918 (2012).

69. Nelson, C. W., Moncla, L. H. \& Hughes, A. L. SNPGenie: estimating evolutionary parameters to detect natural selection using pooled next-generation sequencing data. Bioinformatics 31, 3709-3711. https://doi.org/10.1093/bioinformatics/btv449 (2015).

70. Zuker, M. Mfold web server for nucleic acid folding and hybridization prediction. Nucleic Acids Res. 31, 3406-3415 (2003).

71. Darty, K., Denise, A. \& Ponty, Y. VARNA: interactive drawing and editing of the RNA secondary structure. Bioinformatics 25, 1974-1975. https://doi.org/10.1093/bioinformatics/btp250 (2009).

72. Villordo, S. M., Carballeda, J. M., Filomatori, C. V. \& Gamarnik, A. V. RNA structure duplications and flavivirus host adaptation. Trends Microbiol. 24, 270-283. https://doi.org/10.1016/j.tim.2016.01.002 (2016).

73. Aubry, F. et al. Single-stranded positive-sense RNA viruses generated in days using infectious subgenomic amplicons. J. Gen. Virol. 95, 2462-2467. https://doi.org/10.1099/vir.0.068023-0 (2014).

74. Holm, S. A simple sequentially rejective multiple test procedure. Scand. J. Stat. 6, 65-70 (1979).

\section{Acknowledgements}

The authors thank Pascal Delaunay (Centre Hospitalier Universitaire Nice, France), Ashgar Talbalaghi (Vector Control, Italy), Roger Eritja (Universitat de Barcelona, Spain), Vincent Robert (IRD, Montpellier) for providing mosquito eggs.

\section{Author contributions}

R.B.: acquisition, analysis, and interpretation of data, manuscript revision. S.L.: acquisition, analysis, and interpretation of data, manuscript revision. H.J.: acquisition, analysis, and interpretation of data, manuscript revision. G.P.G.: acquisition, analysis, and interpretation of data, manuscript revision. F.A.: acquisition, analysis, and interpretation of data. L.M.: data acquisition. G.P.: acquisition, analysis, and interpretation of data. P.S.Y.: data acquisition. G.G.: data acquisition. M.V.: data acquisition. A.S.: funding acquisition. G.P.P.: conception and design, manuscript revision. X.D.L.: conception and design. L.L.: conception and design, data analysis and interpretation, writing manuscript. A.B.F.: conception and design, data analysis and interpretation, writing manuscript.

\section{Competing interests}

The authors declare no competing interests.

\section{Additional information}

Supplementary information is available for this paper at https://doi.org/10.1038/s41598-020-75042-4.

Correspondence and requests for materials should be addressed to A.-B.F.

Reprints and permissions information is available at www.nature.com/reprints.

Publisher's note Springer Nature remains neutral with regard to jurisdictional claims in published maps and institutional affiliations. 
(c) (i) Open Access This article is licensed under a Creative Commons Attribution 4.0 International cc) License, which permits use, sharing, adaptation, distribution and reproduction in any medium or format, as long as you give appropriate credit to the original author(s) and the source, provide a link to the Creative Commons licence, and indicate if changes were made. The images or other third party material in this article are included in the article's Creative Commons licence, unless indicated otherwise in a credit line to the material. If material is not included in the article's Creative Commons licence and your intended use is not permitted by statutory regulation or exceeds the permitted use, you will need to obtain permission directly from the copyright holder. To view a copy of this licence, visit http://creativecommons.org/licenses/by/4.0/.

(C) The Author(s) 2020 